\title{
EGREPA 2010 Conference
}

\section{Seniors in the 21 st century - physical activity a tool for health, fitness and social integration}

Prague, September 23-25, 2010

\section{SCIENTIFIC COMMITTEE}

Michael Brach (Germany)

Christoph Breuer (Germany)

Václav Bunc (Czech Republic)

Art Kramer (USA)

Heinz Mechling (Germany)

Yael Netz (Israel)

Michael Sagiv (Israel)

\section{Bio-psycho-social aspects}

The effects of external versus internal focus of attention on a balance task in aging

Reza Abdollahipour

Markazi Province Education Organization, Arak, Iran

\section{Introduction}

Instructions are an important part in teaching new motor skills and include information as to how to perform the skills. The aim of study was to examine whether the learning advantages of an external focus of attention relative to an internal focus, would also be found for the performance and learning of aging.

\section{Methods}

Participants (20 men; age range: 60-70 years) without prior experience were required to practice walking forward on the balance beam. The practice phase consisted of 20 practice trials. One group was instructed to focus on their feet (internal focus), whereas the other group was instructed to focus on the beam (external focus). One day after practice, retention test (5 trials) without instructions was performed. Retention test was consisted of walking forward as fast as possible.

\section{Results}

Although there was no significant difference between external versus internal focus groups in the acquisition phase, both groups showed shorter MTs across trials $P<0.001$. At the retention tests while both groups showed decrease in MTs across trials, $p<00.1$ the external focus group had shorter MTs than the internal focus group, $P<0.05$. Also, the group $\mathrm{x}$ trials interaction was significant, $P<0.05$.

\section{Conclusion}

These results showed that the external focus condition was more effective to perform during both practice and learning. Thus, instructions that directed the performer's attention to the effects that their movements had on the environment (external focus) were more effective on the learning than directing attention to the movements themselves (internal focus) of aging.

Key words: Attentional focus, Movement time, Retention, Aging

References:

1. Abdollahipour, R., \& Bahram, A. (2005). Proceedings of the $4^{\text {th }}$ International Scientific Conference on Kinesiology, Opatija, Croatia, 628-630.

2. Abdollahipour, R., Bahram. A., \& Farokhi. A. (2005). Internal versus external focus of attention effects on transfer to novel situations in the presence of an audience. Proceedings of 9th Sport Kinetics International Conference, Rimini, Italy, 205-207.

3. Abdollahipour, R., Bahram. A., \& Shafizadeh. M., \& Khalaji. H. (2008). The Effects of Attentional Focus on the Learning of a Soccer Dribbling-Task in Children and Adolescences. Journal of Movement Sciences \& Sports, 5 (1), 83-92. 
4. McNevin, N.H., \& Wulf, G. (2002). Attentional focus on supra-postural tasks affects postural control. Human Movement Science, 21, 187-202.

5. Wulf, G. (2007). Attention and motor skill learning. Champaign, IL: Human Kinetics.

6. Wulf, G., Lewthwaite, R., Landers, M., \& Töllner, T (2009). The power of external focus instructions to enhance performance and learning. Physical Therapy, 89, 170-172.

\section{Opinions of young people who participiate to nature youth camps in Turkey}

Zeki Coşkuner, Bilal Çoban, Yüksel Savucu, Sebahattin Devecioğlu, Atalay Gacar

Physical Education and Sports School Firat University, Elazığ, Türkiye

\section{Introduction}

Youth camps spread all over the world. These camps provide many benefits for young people's physically, emotionally and socially developments. But the Turkey's youth camps facilities is very inefficient for young people. Therefore, the number of people is very few. Officially one institution serves to youth camping in Turkey as Youth and Sports Administration (GSGM). The purpose of the present study is to find out perspectives and expectations of the youth who attend as campers in nature camps. The study is to give information about physical conditions of the camps and their practice methods.

\section{Methods}

This study was conducted in Trabzon Sultan Murat Plateau. Between the ages of 18-24, 416 young people participated to the nature camps.

A survey consisting of 32 questions applied to the subjects. The data was analyzed with SPSS for Windows 13.0 program. To analyze the campers' personal information (gender, age, number of camp attendances) frequency methods were used. In determining the camper's perspectives, arithmetic medium and standard deviation methods were utilized. The level of significance for the surveys was examined at 0.05 levels.

\section{Results and discussion}

According to the results of our study;

Campers want more destinations to open the youth camp.

Thanks to this camp, campers have pointed out that the friendship environment better.

Campers want to take advantage of these camps more young people.

\section{Conclusion}

We may conclude that more youth camps should be opened in physical facilities.

Presentation should be made about youth camps well and then ensured the participation of many young people.
Bio-psycho-social Aspects, Chances and Risk-Factors of Everyday Movements in Nursing Home Residents

S. Kalinowski; K. Kopke ${ }^{1}$; I. Wulff; M. Kölzsch ${ }^{2}$; S. Ellert ${ }^{1}$; D. Dräger ${ }^{1}$; R. Kreutz ${ }^{2}$

${ }^{1}$ Institute für Medizinische Soziologie, ${ }^{2}$ Institute für Klinische Pharmakologie und Toxikologie, Charité-Universitätsmedizin Berlin, Germany

\section{Introduction}

Multiple diseases in old age associated with pain are often responsible for impaired motor skills that are necessary in daily routine situations. These basic motor skills are the prerequisite for autonomy, particularly for nursing home residents. In Germany there is a lack of satisfying programs implemented nationwide in nursing homes which facilitate motor abilities. For this reasons, autonomy of institutionalized older adults is even more at risk to be restricted. The purpose of this study is to generate knowledge about personal and environmental aspects promoting everyday movements and autonomy of nursing home residents. This research question extends the research project "Pain and Autonomy in the Nursing home (PAIN)", which is part of the Berlin consortium "Autonomy Despite Multimorbidity in Old Age" (AMA).

\section{Methods}

To explore and identify resources, we focus on internal aspects (physical, psychological, social) and environmental aspects (institutional and socio-economic factors and physical environment) influencing everyday movements. A combination of different instruments was used to obtain primary data: In 41 randomly selected nursing homes in Berlin and Brandenburg, quantitative Data was collected about institutional parameters (manager interview, inspection of the physical environment) and residents $(N=218)$ with face-to-face Interviews, assessments und examination of the institutional documentation.

\section{Results and discussion}

19 nursing homes employ therapeutic stuff. One third put concepts like Bobath into practice. Several aspects demonstrate a scant supply: 11 nursing homes have no individual plans of intervention promoting personal motor skills. 24 homes don't offer courses of instruction for residents and family members concerning capabilities to improve everyday movements. No more than 14 homes offer exercises to prevent falls. Only 5 facilities have ergometers.

One third of the residents state that they don't receive instructions how to improve personal motor skills. Besides they aren't animated. In contrast to the deficient offer, $75 \%$ are motivated. Most offers demanded are non-specific: gymnastics, excursions.

The analysis reveals deficits in communication: Near $60 \%$ of the residents has no sufficient information about proposals of their residence.

Personal and environmental chances to improve everyday movements will be demonstrated. 


\section{Conclusion}

Entire identified resources of the PAIN-Project will help to determine and increase awareness of factors that relate to improved movement abilities and autonomy of nursing home residents.

\section{Comparison of depression rate in retired football and weight lifting athletes}

Mahdi Kohandel, Fardin Fatahi

Islamic Azad University, Karaj Branch, Iran

Introduction: Depression is one of the most common psychological disorders that can happen in different situations such as losing a family member, stress at home or work, and retirement. Post-retirement depression is a common problem among professional athletes. Indeed, making a successful transition from active player to retirement is a major problem for many professional athletes. The athletic career terminations can be generally categorizes as those that are forced (such as de-selection or injury) or freely chosen (increasing age or athlete's own choice). Although the reason of retirement can be important in the study of the post-retirement depression, all professional athletes will be faced with social and physiological changes at the end of their carrier.

The purpose of this study was to determine the depression rate in the retired national team athletes. We studied the effects of several factors such as type of sport, previous history of playing in national team, period of (spending) retirement, and age in the depression rate.

Method: We studied the depression rate in retired football (team sport) and weight lifting (individual sport) athletes. We selected 43 football and 32 weight lifting retired players who have been previous members of national teams with the age range 30-50. We asked them to complete and return the Beck depression questionnaire (created by Aaron $\mathrm{T}$. Beck). The questionnaire had 21 multiple choice questions, which were related to depression symptoms, cognitions, and physical symptoms. The athletes were asked to answer the questions by selecting one of the answers (never, rarely, sometimes, usually, and always) that best fits.

Result/Conclusion: The result indicated that the average post-retirement depression rate is 9.58 in retired football players and 14.47 in retired weight lifting athletes. This clearly shows a significant different between two groups. We also observed a significant correlation between age, period of national team membership, as well as their jobs (if applicable) and the depression rate.

In weight lifting athletes, one of the reasons that create depression may be many of injuries in period of their championship and after retired they can't continue their exercises. Weakness in sport activities probably can be increased depression in retired football player and weight lifting athletes generally.

\section{Resources:}

1. Adams, $m, j$ (1995) psychiatric nursing the hospital and the community. newjersey.oct

2. Antai, Deboruhand kongable Gail (1995) psychiatric nursing biological behavioral concepts, Philadelphia, W.B sanders company

3. Ey,keny,(1984) the psychology of adolescence 4th, Boston, Houghton, miffilin,co

Importance of physical activity in elderly renal patients Andrea Mahrová $^{1}$, Klára Švagrová ${ }^{1}$, Václav Bunc ${ }^{1}$, Vladimír Teplan $^{2}$, Milena Štollová ${ }^{2}$

${ }^{I}$ Faculty of Physical Education and Sports Charles University in Prague, Czech Republic

${ }^{2}$ Institute for Clinical and Experimental Medicine, Prague, Czech Republic

\section{Introduction}

Presented proposal is focused on impact of physical activity on physical fitness, mental condition as well as on the health related quality of the life (HRQOL) in patients with end-stage renal disease (ESRD) on dialysis treatment and after kidney transplantation. For ESRD and dialysis treatment is currently common aging. Patients often suffer from musculoskeletal system disorders affecting functional ability. HRQOL is reduced in sphere of self-sufficiency, following with limitations in psychosocial area. Regular physical activity is found to be accompanied by distinct physical and psychosocial benefits for them.

\section{Objectives}

To evaluate the actual status of physical and mental condition in elderly dialysed and renal transplant patients. To apply regular exercise program within and outside dialysis and evaluate its impact on functional and psychosocial condition.

\section{Methods}

We observed two groups of renal patients. Group A: patients on hemodialysis -males $N=34$ (mean age 67,0土 12,7 yrs); females $N=33$ (mean age $64,0 \pm 13,1$ yrs). Group B: patients after successful kidney transplant (November 2009-June 2010). Physical condition we evaluated with "Senior Fitness Test-SFT". HRQOL we evaluated with standardized questionnaire SF36-Bref. Firstly we compared results with population norms and secondly after an exercise program (pre-post-tests).

\section{Results}

Generally, physical and mental condition was decreased in comparison with non-renal individuals. Group A: in physical tests in female's group we found the only statistically significant change in post- exercise programme performance 
for Sit to stand test $(p=0,04)$. In the other SFT tests was found at least $50 \%$ improvement in four out of seven tests. Among group of male's there were not found statistically significant changes. At least a 50\% improvement was observed in four out of seven tests. In SF36-Bref was found a strong improvement in HRQOL components: RE-Emotional limitations of Roles and EV-Vitality. Group B: results are in evaluation process. In comparison with normative standards we presuppose their insufficient physical and mental fitness.

\section{Conclusions}

The positive influence of regular physical activity on the physical fitness and HRQOL was confirmed. An acceptable choice of regular physical activity together with other treatment methods seems to be good way of nonpharmacological character, that offers to the elderly renal patients an opportunity to achieve an optimal functioning and psychosocial level very important for long-term maintaining of self-sufficiency and independence.

This study was supported by the projects: GACR 406/07/P443; MSM 0021620864; IGA MZ ČR 173 (NS-10518-3/2009).

\section{Enjoying locomotion activity in old age Miroslav Štilec, Václav Bunc}

Youth and locomotion activity - primary motivation, pleasure in locomotion, performance-all these are generally known terms.

The concept of finding pleasure in locomotion in senior age, however, still waits for its full exploitation and recognition. It lacks importance, which is also affected by a sceptical attitude of society towards old age.

Economically advanced countries where systematic care of senior citizens has been running for min. 15-20 years report a $15-20 \%$ interest on the part of adult and elderly populations who participate in regular preventative healthoriented exercises. At the same time, however, open literature states that within half a year $50 \%$ of those interested quit without objective reasons.

Psychologists such as Dishman, Hošek and others claim that loyalty as persistence - adherence to regular exercises in elderly people is supported not only by a mere awareness of good health and longevity i.e. by rational motivation. People, quite rightly, also want to gain emotional experiences from locomotion activity, enjoy the atmosphere and sociable spirit associated with locomotion. This, however, is not respected in the locomotion programme for elderly people, and the creation of joyful and entertaining atmosphere that strengthens the will to persist is not adequately targeted.

The experience from our project confirms that, except for individuals with major health handicaps, people do not quit without reasons, on the contrary, new adepts ask to be included in the exercise programme.

Perception in old age is of specific nature, involving in particular the awareness of one's own body, gaining confidence in one's own body, confidence in one's acts, conduct. By enhancing the perception of one's own body, we gain or regain the experience that locomotion may be a source of joy and internal feelings enriching a person regardless of age.

Our project whose output is presented in the article was conceived as an intra-group survey focused on monitoring the changes and development of individuals within a group. The intention was to assess cross-sectional investigations (functional, motor, psychosocial) and the effect of exercises aimed at enjoying locomotion on the lifestyle and feelings of personal independence of seniors. The surveyed group was constituted by addressing Prague's citizens aged over 60.

The project programme was conceived as a balanced unit in terms of physical and mental load.

The results of the tests and the final self-appraisal by the seniors confirmed that the involution phenomena had been markedly suppressed in the functional, motor and as well as mental area. Positive results were also evident in the locomotion fitness, dexterity and enhanced confidence of the seniors in their own abilities. They lived more joyful lives knowing that they are able to react to their life circumstances more flexibly; the same was also perceived by people in their close surroundings.

Influence of square stepping exercise on executive functions and attention in older people

${ }^{1}$ Camila Vieira Ligo Teixeira, ${ }^{1}$ Sebastião Gobbi, ${ }^{1}$ Jessica Rodrigues Pereira, ${ }^{1}$ Deysi Terumi Ueno, ${ }^{1}$ Thays Martins Vital, ${ }^{1}$ Salma S. Soleman Hernández, ${ }^{2}$ Ryosuke Shigematsu, ${ }^{1}$ Lílian Teresa Bucken Gobbi

${ }^{1}$ Instituto de Biociências, UNESP-Univ. Estadual Paulista, Laboratórios de Atividade Física e Envelhecimento (LAFE) de Estudos da Postura e Locomoção (LEPLO), Rio Claro-SP, Brazil; ${ }^{2}$ Faculty of Education, Mie University, Japan.

Introduction: Cognitive functions may not always decline with aging but they are affected by several factors such as social environment and lifestyle. Several studies have demonstrated that physical exercise has positive influence on cognitive functions, including executive functions (EF) and attention (AT). Square Stepping Exercise (SSE) is a relative novel exercise mode, which was primarily designed for falls prevention in the elderly (Shigematsu \& Okura, 2006). However, it is possible that SSE may also improve cognitive functions. Therefore, the aim of this study was to 
analyze whether SSE intervention benefits executive functions and attention in older people.

Methods: The participants were 21 community-dwelling subjects, aged 60 to 92 year-old $(68.47 \pm 8.37$ year-old), who were enrolled in 45-min training sessions of SSE, 3 times per week, during 4 months. The intervention was developed according to the SSE sequences solely, that is, without addition of any warm up's, cool downs, aerobic or resistive exercise. At pre- and post-intervention, the Modified Card Sorting Test and Tolouse test were applied to assess the executive functions and attention, respectively. Wilcoxon test was chosen to compare the pre- and postresults.

Results and Discussion: After 4 months of intervention there were significant improvements $(p<0.05)$ on EF (26.56 \pm 8.01 and $12.73 \pm 10.17$ preservative errors) and on AT $(59.13 \pm 33.36$ and $73.86 \pm 45.27$ correct trials), pre and post-intervention, respectively. Although, searching for mechanisms was out of scope of the study, SSE intervention may have benefit executive functions and attention due to the cognitive demand it requires to perform the pattern of its sequences. This may have happened in conjunction with potential beneficial changes due to physical exertion itself. Conclusions: Intervention by means of Square Stepping Exercise seems to improve executive functions and attention in older people.

References: SHIGEMATSU, R., OKURA, T. A novel exercise for improving lower-extremity functional fitness in the elderly. Aging Clinical and Experimental Research. Vol 18, n 3, p. 242-248, 2006.

Acknowledgments: CNPq; FUNDUNESP; PROEX-UNESP.

\section{Physical activity}

An analysis study describing the factors affecting cognitive function in older adults: a Turkish sample Ugur Cavlak, Emine Aslan Telci, Beyza Akdag

Introduction: Cognition can be thought of in terms of function of the brain, such as memory, association, (verbal and quantitative), spatial ability and manipulation, and synthesis. The processes of cognition- attention (mental energy), working memory, information-processing speed, psychomotor ability, and perception- support to cognitive functions. The purpose of this study was to determine the influential factors of cognitive function of the elderly living Denizli, Turkey.

Methods: Three hundred and seventy seven older adults (91 living in a rest home; 286 living in their home) with a mean age of $74.7 \pm 0.3$ years (range; 65-94 yr.) were evaluated in the study. The Hodkinson Mental Test was used to exam cognitive function of the sample. Also, the influential factors of cognitive function were detected. To determine the influential factors of cognitive function (Hodkinson Mental Test Score), the Binary Logistic Regresion Method was used. In statistical analysis, age, gender, occupation, education level, environment, smoking habit, musculoskeletal pain (last 3 months), medication, emotional status (last 1 month), and number of physically unhealthy days (last 1 month) and activity limitation (one last month) were studied.

Results and Discussion: The results of the Binary Logistic Regression Method showed that age, occupation, environment, emotional status (last 1 month) was found to be influential factors of the Hodkinson Mental Test score. Age was found to be the most affecting factor cognitive function in the sample. The following parameters were also found to be affecting factor cognitive function; (1) to be housewife; (2) living in a rest home; (3) number of mentally unhealthy days.

Conclusion: Cognitive function of the elderly participants living in Turkey was seen to be affected by age, occupation, environment, and emotional status.

\section{References:}

1. Anstey K.J., Low L.-F. - Normal Cognitive Changes in Aging. Aust Fam Physician 2004; 33 (10): 783-787

2. Dirik A., Cavlak U., Akdag B. - Identifying The Relationship Among Mental Status, Functional Independence and Mobility Level in Turkish Institutionalized Elderly: Gender Differences. Arch Gerontol Geriatr. 2006; 42(3): $339-50$

3. Avila R., Moscoso M.A.A., Ribeiz S., et al. Influence of education and depressive symptoms on cognitive function in the elderly. Int Psychogeriatr. 2009;21(3):560-7.

4. Andel R., Kåreholt I., Parker M.G., et al. Complexity of Primary Lifetime Occupation and Cognition in Advanced Old Age. J Aging Health. 2007: 19 (3): 397-415

5. Gruber- Baldini A.L., Zimmerman S., Boustani M. Characteristics Associated with Depression in Long-Term Care Residents with Dementia. Gerontologist. 2005; 45: $50-55$.

Concept and prototypical realisation of an accelerometrybased integrated fall detection and prevention system for the Elderly

M. Daumer ${ }^{1,2}$, A.Schlummer ${ }^{1}$, M.Schimpl ${ }^{1}$, C. Soaz ${ }^{1}$, A. Kokkalis ${ }^{1}$, A. Neuhaus ${ }^{1}$

1 Sylvia Lawry Centre-The Human Motion Institute

2 Trium Analysis Online GmbH

\section{Introduction}

About $35-40 \%$ of the elderly $>65$ years and $50 \%$ of $>80$ years fall at least once a year [1]. A major problem is that half of the 
elderly who fall at home become helpless and require assistance to get up. That could be avoided by automatically calling for help when a person was unable to summon help [2]. Current commercially available alarm systems suffer from problems with usability and high false alarm rates.

\section{Objectives}

To develop a prototype of an accelerometry-based system for fall detection with high user acceptance, high sensitivity and low false alarm rate linked to an evidence-based decision support tool for fall prevention.

\section{Methods}

The actibelt(R), a ICT-platform including a tri-axial accelerometer integrated in a belt buckle, was developed [3]. Threshold-based algorithms for fall detection and gait speed were developed and validated. In parallel a biomedical data warehouse for physical activity data is built.

\section{Results and Discussion}

An independent evaluation of the usability of actibelt(R) showed its high user acceptance [4]. This was confirmed in a recent study with 357 healthy people (mean age: 48.1, sd 12.3): $78 \%$ of the participants wore the actibelt(R) over the whole study time ( 7 days), $88 \%$ for at least $85 \%$ of the study time. Fall detection algorithms were tested using data from "artificial" falls. Sensitivity and specificity were $80.6 \%$ and $63.3 \%$, resp. False alarm rate was estimated from long-term measurements ( $>1$ week, $n=19)$ with 1.2 / $24 \mathrm{~h}$. Gait speed assessment based on support vector regression has a mean error rate of $8 \%$ and a concordance correlation coefficient of $95 \%$.

\section{Conclusion}

First steps to integrate mobile accelerometry into a fall detection system are taken. Further refinement and validation of the fall detection algorithms is necessary to reduce false alarm rates. Prospective trials in seniors will be conducted to explore early warnings for falls that can then be integrated in a system for fall prevention. Decrease of gait speed is of particular interest since it is a predictor for health-related events such as falls [5].

\section{Literature}

[1] Gostynski M. Prevalence, circumstances and consequences of falls in institutionalized elderly. Soz Praventivmed. $1991 ; 36(6): 341-345$

[2] Campion E. Home alone and in danger. N Engl J Med 1996,334:1738-9

[3] Daumer M. et al, Biomed. Technik/Biomedical Engineering 2007,52:149-55

[4] Scheermesser M. et al. User Acceptance of Pervasive Computing in Healthcare: Main Findings of two Case Studies. Pervasive Computing Technologies for Healthcare, 2008,205-213

[5] Purser J. et al. Walking speed predicts health status and hospital costs for frail elderly male veterans. J of Rehabilitation Research \& Development. 2005,42:535-546
Physical exercise, daily physical activity and blood lipid profile in post-menopause

Andrea Di Blasio, Francesco Di Donato, Ines Bucci, Angela De Stefano, Giorgio Napolitano, Patrizio Ripari Department of Human Movement Sciences, "G. D'Annunzio" University, Chieti-Pescara, Italy

\section{Introduction}

Despite a number of review article summarize the positive effect of physical exercise on the blood lipid profile, sometimes both literature and clinical practice report the inefficacy of physical exercise to improve plasma lipoprotein levels. Because of some studies indicated that the positive effect of physical exercise on blood lipid profile is related to its amount but not to its intensity, we investigated whether a right intervention should only control the physical exercise program or both physical exercise program and daily physical activity.

\section{Methods}

Forty-one sedentary postmenopausal women were enrolled for the study and were evaluated before and after 14 weeks of aerobic training. Blood was collected to measure out plasma levels of triglycerides, total cholesterol (TC) and both low and high-density lipoproteins (LDL, HDL). Anthropometry and body composition analysis included the measurements of body weight and height, waist and hip circumferences and single frequency electrical bioimpedance. Dietary habits were estimated by a dietician using the WinFood 2.7 software (Medimatica). Maximal stress test gave the eligibility to exercise training and the maximal aerobic fitness (VO2max). Participants walked for $50 \mathrm{~min}$, 4 days/wk, at moderate intensity. Participants' physical activity were measured through SenseWear Armband (Bodymedia). It gave information about total daily energy expenditure (TEE), mean daily physical activity intensity (METs $\mathrm{m} / \mathrm{die}$ ) and both time and energy spend on physical activity with intensity $>3$ METs (tPA, PAEE). Measurements lasted 3 consecutive days and were repeated 20 days before to the end of the training $\left(T_{1}\right) . T_{1}$ measurements included 1 training day and 2 resting days. Statistical analysis was performed on the 35 participants $(55.60 \pm$ $4.11 \mathrm{yrs})$ free from medications.

\section{Results and discussion}

Paired Sample t-Test showed that aerobic training elicited only the increase of VO2max $(p<0.001)$. In order to study the absence of TEE, METs $\mathrm{m} / \mathrm{die}$, tPA and PAEE significant variations, cluster analysis was performed on their delta and detected two groups with the same adherence to training: one (GROUP + ) improved its spontaneous physical activity while the other reduced that (GROUP-). RM-ANCOVA showed that GROUP+ increased HDL and reduced LDL and TC while GROUP- had opposite results. Both of them had the same variation of VO2max. 


\section{Conclusion}

Our results confirm that, while physical exercise intensity and duration are important for VO2max improvement, the amount of daily movement is important for lipoproteins control and suggest the necessity to monitor spontaneous physical activity when the major target is the blood lipid profile improvement.

\section{Multimodal exercises improve gait and clinical} parameters of Parkinson's disease patients

Lilian T. B. Gobbi ${ }^{1}$, Rodrigo Vitório ${ }^{1}$, Claudia TeixeiraArroyo $^{1}$, Ellen Lirani-Silva ${ }^{1}$, Fabio A. Barbieri ${ }^{1}$, Maria J. D. Caetano ${ }^{1}$, Florindo Stella ${ }^{1,2}$, Sebastião Gobbi ${ }^{1}$

${ }^{1}$ UNESP_Univ Estadual Paulista, Rio Claro, SP, Brasil.

${ }^{2}$ UNICAMP_Campinas State University, SP, Brasil

\section{Introduction}

Gait disorders are one of the most incapacitating signs of Parkinson's disease (PD), and exercise trials have been shown to be effective in improving gait parameters (Goodwin et al., 2008). Additionally, the literature suggests that in exercise trials it is crucial for investigators to consider effects on clinical symptoms in conjunction with changes in gait (Sage, Almeida, 2009). Therefore, the aim of this study was to identify the effects on PD patients of a multimodal exercise program on gait kinematic and clinical parameters.

\section{Methods}

Twenty-one patients with mild to moderate idiopathic PD participated in the study. They were assessed before and after enrollment in a long-term, multimodal exercise program that focused mainly on aerobic endurance. The program took place over a six-month period ( 72 sessions, 3 times a week, and 60 min per session). A more detailed description of the training protocol was published by Tanaka et al. (2009). Outcome measures included the subsections of the Unified Parkinson's Disease Rating Scale (UPDRS) and the spatiotemporal parameters of self-paced walking. The data (not normally distributed) were treated by the Wilcoxon non-parametric test for two related samples (pre- and post-test).

\section{Results and Discussion}

In relation to clinical data, the PD patients' scores on the UPDRS-II decreased after participating in the program $(Z=-2.167 ; p=0.03)$. With regard to the gait dependent variables, PD patients increased stride length $(Z=-4.402 ; p \leq 0.001)$, stride velocity $(Z=-5.732 ; p \leq$ $0.001)$ and cadence $(Z=-5.302 ; p \leq 0.001)$, and decreased stride duration $(Z=-5.548 ; p \leq 0.001)$, after participating in the program. Petzinger et al. (2010) indicated that the modulation of cortical hyper-excitability is the underlying mechanism for exercise-induced behavioral improve- ments in animal models of PD. Exercise intervention leads to compensatory changes in dopamine handling and neurotransmission.

\section{Conclusion}

The multimodal exercise program was effective in promoting an improvement in clinical and gait kinematic parameters for patients with mild to moderate PD.

\section{References}

Goodwin VA et al. (2008). Movement Disorders;23:631-40. Petzinger GM et al. (2010). Movement Disorders;25:S141-5. Sage MD, Almeida QJ (2009). Movement Disorders; 24:1132-8.

Tanaka K et al. (2009). Brain and Cognition;69:435-41. Acknowledgements: FAPESP, CNPq, CAPES.

Comparison the effects of Hydrotherapy, massage and stabilization training on the Chronic low back pain in the men personal ahvaz oil company

Siros Hosseini ${ }^{1}$, Roholla Valizadeh ${ }^{2}$

${ }^{1}$ Islamic Azad University, behbahan Branch

${ }^{2}$ Islamic Azad University, omidieh Branch

\section{Introduction}

Exercise is one of the most important treatment that is prescribed with low back pain which has been demonstrate effect on improvement of function an work of patients in this study for the first time comparison of the effects Hydrotherapy, massage and stabilization training on the Chronic low back pain had been survey.

\section{Methods}

The purpose of the present study was to assess the comparison of the effects Hydrotherapy, massage and stabilization training on the Chronic low back pain in the men personal ahvaz oil company. Sixty men with Chronic low back pain, aged 30-40 years old, voluntarily participated in this study. Pain of subjects were assessed by Q-back questionnaires respectively. All measurements on patients were respected after 12 weeks exercise. Analyses of data by using ANOVA showed that pain back decreased after treatment

\section{Results and Discussion}

The results of this research show that hydrotherapy and stabilization training had positive and significant effect ( $p$-value $=0 / 000)$ on the Chronic low back pain that this effect was strong for hydrotherapy exercises. Also comparison between massage and control groups showed that there is no signification different between them. According to studies seem that with increase of exercise time, the massage training can be effect on low back pain but in this research seem that 8 weeks exercise was not enough for massage group. on based before studies regular training program can be help to decrease low 
back pain. But about massage exercises we do not have enough studies available $(1,2)$

\section{Conclusion}

This result and all of the research like it had shown that all of the people in the world that have pain back, they need do exercise. But which exercise can be better than another. Because that some exercises harmful for pain back. Hydrotherapy and stabilization training are tow kind of the them that very useful for pain back.

\section{References}

1-Furlan AD, Imamura M, Dryden T,(2009).Irvin E Massage for low back pain: an updated systematic review within the framework of the Cochrane Back Review Group. Institute for Work \& Health, Toronto, ON, Canada. Publication Types, MeSH Terms Spine, (16) 1669-84

2-Tsao JC(.2007). Effectiveness of Massage Therapy for Chronic, Non-malignant Pain: A Review. Pediatric Pain Program, Department of Pediatrics, David Geffen School of Medicine at UCLA, USA. Evid Based Complement Alternat Med, 4(2) 165-79

The study of Relation between different levels of maximal oxygen uptake and tracheal airway obstruction in men personal in N.I.S.O.C

Siros Hosseini ${ }^{1}$, Roholla Valizadeh ${ }^{2}$

${ }^{1}$ Islamic Azad University, behbahan Branch

${ }^{2}$ Islamic Azad University, omidiyeh Branch

\section{Introduction}

Today the most of the people in the word know that active men have successful life than inactive person and activity must be take maximal oxygen uptake that one of the important way for show airway obstructive.(1)

\section{Methods}

The aim of this study was evaluation of relation between different level of maximal oxygen uptake and tracheal airway obstruction. The men in this between $40-50$ years and $171.94 \mathrm{~cm}$ median length and $37 / 37 \mathrm{ml} / \mathrm{kg} / \mathrm{min}$ aerobic capacity. First we select 1,200 cases of men personal and then select 350 number of this cases that have a our criteria. finally randomly, select three groups that any groups have a 45 members . this three group divided to mild, moderate and sever according to maximal oxygen uptake that take by use of cooper test on treadmill this cases have a complete health with out any physical disease specially pulmonary disease .analyses of data by using ANOVA and LSD test

\section{Results and Discussion}

The finding in this research showed that there is significant different with $(p$-value $=0 / 000)$ between many pulmonary index (FVC, FEV1,FEV1/FVC) and 3 levels of maximal oxygen uptake (mild, moderate, strong) that
With increase of maximal oxygen uptake(vo2max) the airway obstruction will be decrease. And another result show that with increase amount of vo2max the pulmonary index (FVC, FEV1,FEV1/FVC) will be increase too. According to the results of this investigation we can say that maximal oxygen uptake can be effect on airway abstraction and decrease it. The results of this research in harmony with before studies that suggested the active persons that they have most maximal oxygen uptake (vo2max), they have lower airway obstruction compared with suddenly people $(2,3)$.

\section{Conclusion}

Based on this result and all of the research like it, has been shown that active people and athlete have high pulmonary index than inactive people because the active people have been strong breathe muscle, lower fat and higher (von2max). Also activity can be help to people that they have low pulmonary index.

\section{References}

1-Yue Chen and et al (2007) Waist circumference is associated with pulmonary function in normal-weight, overweight, and obese subjects, 85(1), 35-39

2- Brydon J.B (2006). Pulmonary Function and Abdominal Adiposity in the General Population. Chest, 129,853-862 3-Jones RL (2006). The effects of body mass index on lung volumes, 130(3) 827-33

Effect of aging on skill relate fitness in Iranian women Kordi Mohamad Reza ${ }^{1}$, Rezaei Nejad Najmeh ${ }^{2}$, Shirafkan Maryam $^{2}$

${ }^{1}$ Faculty of Physical Education and Sport Sciences, University of Tehran, Tehran, Iran

${ }^{2}$ Faculty of Physical Education and Sport Sciences, University of Alzahra, Tehran, Iran

Introduction: Human physical performance is notably reduced with ageing (1).

Loss of muscle strength and physical fitness as a result of aging is reported to impair functional ability in various communities. Power deteriorates at a faster rate than strength with advancing age. Physical activity level at baseline was associated with lower risks of disease and dementia of any type 5 years after assessment. The aim of this study was investigate the effect of aging on skill relate fitness in Iranian women (20-60 years).

Material: A sample of 1000 Iranian women aged 2060 years in 4 age groups $\{n=250(20-29 y r), n=350$ (30$39 \mathrm{yr}), n=250(40-49 \mathrm{yr}), n=150(50-59 \mathrm{yr})\}(\mathrm{M} \pm \mathrm{SD}: 158 \pm$ $0 / 05 \mathrm{~cm}, 68 / 78 \pm 9 / 6 \mathrm{~kg}$ ) volunteered to participate in this study. All subjects were originally from different parts (north, south, west, and east) of Tehran. The agility of subjects measured with the up and go test. For measured of 
speed use of 20 meters running test, and for balance use of stroke test, and power measured with sergeant test.

Results: The results of this study showed that there is significant different in agility $(p=0 / 00)$, power $(p=0 / 00)$, balance $(p=0 / 00)$ and speed $(p=0 / 00)$ between 4 age group. Conclusion: The result showed with increase in age the skills relate fitness was lower. The changes in functional ability have a significant relationship to aging (2). Physical fitness and nutritious diet is the key for longevity and to hide the signs of old age. Recent studies, however, have suggested that selective atrophy of type II fibers may be more related to disuse rather than ageing, since it can be reversed by resistive training(3).

\section{Reference:}

1. Jörn Rittweger, Pietro Enrico di Prampero2, Nicola Maffulli (2009).Sprint and endurance power and ageing: an analysis of master athletic world records. Proc R Soc B, February 22, 276(1657): 683-689.

2. Kramer. A. F., Stanley J. C, Edward M, Paige E. S, Kirk I. E.(2005). Fitness, aging and neurocognitive function. Neurobiology of Aging 26S-S124-S127.

3. di Prampero P.E (2003). Factors limiting maximal performance in humans. Eur. J. Appl. Physiol. 90, 420-429

Selected coordination indicators of the inclusion of shoulder girdle muscles during nordic walking Bronislav Kračmar, Radka Bačáková, Petra Tlašková Faculty of PE and Sports Charles University, Prague, Czech Republic

\section{Introduction}

A high percentage of the old who are physically relative fit can be go in Nordic Walking (NW). During walking with poles the work of lower limbs is in fact the same as during common fast walking and the work of trunk and upper limbs could be assimilated to the movement during classical technique in cross-country skiing. Human locomotion is realized by the pelvis girdle, the shoulder girdle have then humanization function, function of manipulation and grip. The shoulder girdle is a dominant structure realizing locomotion of a child in the human locomotion phase called spontaneous crawling or seal movement. The locomotion function of shoulder girdle becomes then in the fourth trimester the secondary function.

\section{Sample}

The sample consisted out of 6 healthy tested persons, without subjective difficulties, 4 women, 2 men during 4 measurements, aged 22-53, teachers of cross-country skiing.

\section{Methods}

At each tested person we have analyzed 8 times $20 \mathrm{~s}$ of each motor activity. Measurement of each activity was evaluated 5 times. Intra-individual comparative analysis of free bipedal walking and NW is framed on the basis of the EMG observation with the synchronized video record. We have used the portable measuring device working on the basis of EMG potentials, carried on the body of the tested person. Synchronization in video sequences was realized by the software Darfish.

\section{Results}

Figure 1 - Selected double step of the tested person No 4 NORDIC WALKING

FREE WALKING

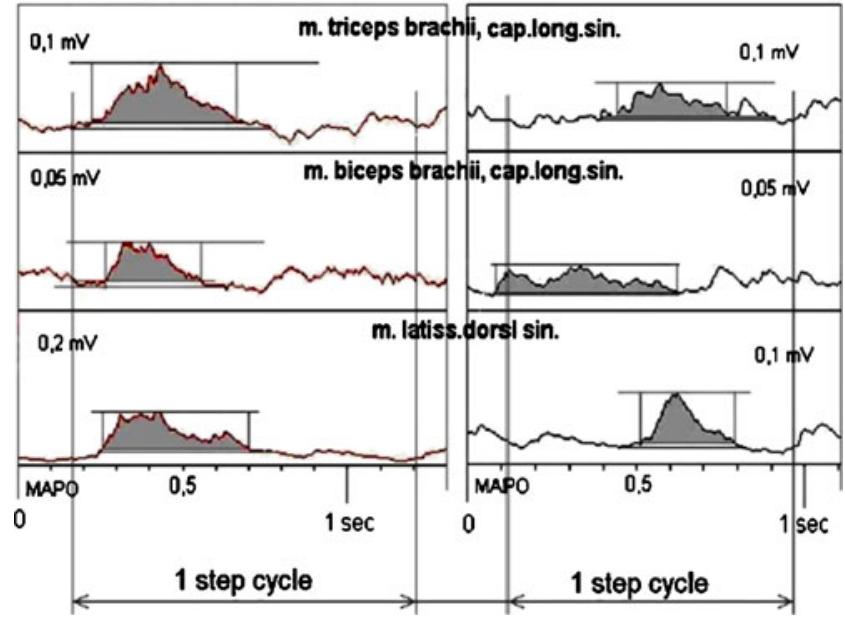

Table 1 Average correlation coefficients of mutual muscle timing in all 6 tested persons during free walking and $\mathrm{NW}$

\begin{tabular}{|c|c|c|c|}
\hline \multicolumn{4}{|c|}{ Nordic walking } \\
\hline & m.latiss. & m.tric.br. & m.bic.br. \\
\hline m.latiss. & $x$ & 0,936 & 0,921 \\
\hline m.tric.br. & 0,936 & $x$ & 0,897 \\
\hline m.bic.br. & 0,921 & 0,897 & $x$ \\
\hline \multicolumn{4}{|c|}{$\begin{array}{l}\text { percentage expressing average standard } \\
\text { deviation in all tested pers. } \quad 14,2 \%\end{array}$} \\
\hline \multicolumn{4}{|c|}{ Free walking } \\
\hline & m.latiss. & m.tric.br. & m.bic.br. \\
\hline m.latiss. & $x$ & 0,675 & 0,269 \\
\hline m.tric.br. & 0,675 & $x$ & 0,512 \\
\hline m.bic.br. & 0,269 & 0,512 & $x$ \\
\hline \multicolumn{3}{|c|}{$\begin{array}{l}\text { percentage expressing average standard } \\
\text { deviation in all tested pers. } \quad 41,8 \%\end{array}$} & \\
\hline
\end{tabular}

Figure 2 shows the selected section of EMG graphs of the tested person D. P. during NW and free walking. Table 1 shows average correlation coefficients of muscle timing in all six tested persons.

\section{Discussion}

We can see very interesting interrelations of cooperation of the observed muscles. In all tested persons we have found very high correlation coefficients in the observed step cycles during NW, around values 0.9 . However, a very low correlation between working phases of muscles was found during free walking, between $\mathrm{m}$. latissimus dorsi and $\mathrm{m}$. biceps brachii, cap.longum even under the level 0, 3 . 


\section{Conclusion}

The change of muscle coordination in the regime of phasic work for balancing torsion impulses during free walking into the regime of phasic work for creating propulsive strength to the new formulated punctum fixum (operationally formulated as a locomotion regime of work) was confirmed in all six tested persons.

We can conclude that walking with poles could be for old and oldest a suitable motor activity mainly for the area of shoulder girdle serving for activation of original movement patterns of the shoulder area-for example as a complement after the finished basic rehabilitation of this segment.

The research was realized thanks to the grant support GAČR 406/08/1449 and GAUK 64907/2007 and was supported by grant of Czech Ministry of Education MSM 0021620864.

\section{Influence of blood pressure on changing postural stability after moderate effort in elderly men \\ Janusz Maciaszek, Rafał Stemplewski \\ University School of Physical Education in Poznań, Poland}

The aim of the study was to assess a range of postural stability changes after moderate effort in elderly people depending on systolic blood pressure.

The study included 27 men aged 65 to 81 years $(71.6 \pm 4.9)$. Subjects had their SBP measured in the sitting position. Postural stability was tested before the effort and directly after its completion. Postural sway (PS) during bipedal quiet stance was assessed. The shifts in vertical projection of the centre of pressure on the platform (COP) were collected using the AccuGaitTM System posturographic plane (AMTI PJB-101 model, AMTI, Waterdown, MA). The following parameters related to COP sway were taken into consideration: Path length (mm) - total length of COP sway, Area95\% $\left(\mathrm{cm}^{2}\right)$ sway area limited with an ellipse of the 95th centile.

After the first measurement of postural stability the subjects carried out physical effort for $10 \mathrm{~min}$ on cycloergometer under a constant medical supervision. The level of intensity of the effort was established on the basis of heart rate (HR). The target values of training HR was calculated on the basis of the Karvonen formula (1957). The target value of training HR was calculated for $60 \%$ of $\mathrm{VO} 2 \mathrm{max}$.

Our study of elderly men showed that rectilinear relation describes to a small extent the relations between SPB in rest and post-effort change in path length $\left(R^{2}=5.3 \%\right)$ as well as between SBP and area95\% $\left(R^{2}=10.8 \%\right)$. The relations between resting SBP and change in postural stability after effort are curvilinear and adopt the shape of a parabola. Relations described in this way have the value of $R^{2}=24,1 \%$ for the relation between SBP and path length and $R^{2}=36.4 \%$ for the relation between SBP and area95\%.

Using only methods of determining straight relations in statistical analyses may lead to significant simplifications and to drawing biologically wrong conclusions which have serious practical consequences. The smallest post-exercise changes in postural stability are noted in people with resting $\mathrm{SBP} \approx 130 \mathrm{~mm} \mathrm{Hg}$. Lower or higher values of SBP are related to the risk of increasing the path length and area $95 \%$, that is temporary decrease in postural stability.

The elderly who love to fall-Sport parachuting $\&$ its health implications in the elderly

Abhishek Mathur

Specialty Registrar, Care of the Elderly, University Hospitals of Leicester NHS Trust, UK

\section{Introduction}

Skydiving or sport parachuting is an adventure sport in which jumpers exit an aircraft at an altitude of $1-4 \mathrm{kms}$ above ground level and fall freely for a brief period before deploying the parachute. Though mostly popular with the young, an increasing number of the elderly, sometimes frail, have indulged in this sport, often with resulting media coverage.

\section{Background}

Indulging in such an adventure sport by the elderly is often an expression of challenging limitations, proving a point, or even a celebration of "being alive". However, there are health implications which are expectedly different and more serious in the elderly as compared to the younger jumpers. A host of physiological changes occur when the jump is made from an unpressurised aircraft without supplemental oxygen. The presence of cardio-respiratory pathology and its severity needs to be assessed in order to establish relative risks involved with the physiological stresses. Similarly, Musculoskeletal and Neuro-psychiatric fitness need to be assessed.

\section{Methods}

Material relating to health implications in sport parachuting from peer-reviewed journal publications, print media and internet reports, particularly those related to jumps made by the elderly was reviewed. The literature of the British Parachuting Association, including policy documents relating to assessment of fitness to jump and injury data was also reviewed. Contact was made with 26 British clubs affiliated to the British Parachuting Association to ascertain if safety or injury data was being compiled for the elderly and what processes were in place for establishing fitness to jump.

\section{Discussion}

Adventure sports such as sport parachuting are being tried by the elderly for a variety of reasons. These sports have 
inherent hazards which become more pronounced in the elderly due to lower physiological reserves and susceptibility to trauma and serious injury. The assessment of fitness, for such activities in the elderly, needs to be robust, in order that adverse events can be avoided.

\section{Conclusion}

Assessment methods for fitness of the elderly intending to participate in adventure sports needs to be standardised. There needs to be better collection of data relating to performance, safety and injury with regards to the elderly who participate in adventure sports.

The effects of a strength training programme upon strength and flexibility in the upper-limbs of previously sedentary elderly women

Piccinno, A; Sannicandro, I.; Rosa , R.A; Sperinteo C

University of Foggia, Italy

\section{Introduction}

Aging is characterised by reductions in muscle mass and consequent reductions in muscle strength that vary according to gender (1). Assessments of strength and flexibility are important because these two capacities are correlated to prolonged physical autonomy in the elderly (2) The objective of the study was to assess the effects of a strength training programme upon strength and flexibility in a sample of previously sedentary elderly women.

\section{Methods}

72 elderly women (mean age +/-SD: $71.3 \pm 3.2$ ) were divided into two groups: EG $(n=16)$ and $\mathrm{CG}(n=16)$.

The EG carried out the following own-body weight strength training arm exercises and exercises involving $2 \mathrm{Kg}$ dumbbells: alternate arm-curls, simultaneous arm curls, wall pushups with varying arm widths, side lateral raises, sideward inclinations of the torso, a spine twist, and upward arm stretches from a seated position (1 series of 8 repetitions).

The CG carried out recreational motor activities (ballroom dancing, walking); activities executed were not quantified.

The training period was 12 weeks with bi-weekly training sessions.

Strength and flexibility of the upper limbs were assessed using the Arm Curl Test and the Back Scratch Test respectively (3)

\section{Results and Discussion}

The results are summarized in table 1 .

\begin{tabular}{lllll}
\hline & EG pre- & EG post- & CG pre- & CG post- \\
Arm Curl Test & $17 \pm 2.8$ & $21 \pm 3.8 \# \# \#$ & $18 \pm 2.4$ & $18 \pm 2.5^{*}$ \\
Back Scratch Test & $-6 \pm 7.9$ & $-3 \pm 7.4$ & $-8 \pm 9.9$ & $-5 \pm 9.3$ \\
\hline
\end{tabular}

* inter-group difference \# intra-group difference
Despite the brevity of the training programme, the results show improved EG performances in upper-limb strength, although only to a limited extent. No significant changes in flexibility (a capacity that requires focused training in the elderly) were observed; the post-training increments in flexibility were similar between the two groups.

\section{Conclusion}

The considerations relative to the proposed training protocol could indicate the necessity to stipulate specific activity relative to flexibility in the sedentary elderly women: the training protocol was dedicated to strength training did not produce any consistent and efficient improvements in mobility and flexibility (4); however, this training was still found to exclusively oppose the effects of the aging process.

\section{References}

Lemmer, J.T., Martel, G.F., Hurlbut, D.D., Hurley, B.F. (2007). J Strength Cond Res 21(3):731-737

Pereira, F.F., Monteiro, N., Vale, R.G.S., Gomes, A.L.M., Rev Esp Geriatr Geront, 42(6),319-324.

Rikli R.E., Jones C.J. (1999). J Aging Phys Act , 7 : 162-181. Sannicandro I., Colella D., Rosa A.R., Manno R., Medicina dello Sport, 61: 443-454. 2008

Effect on the amateur footballer's blood lipids of quick strength and aerobic endurance trainings

Yüksel Savucu*, Abdülkerim Hamzaoğulları**, Hüseyin N. Özaltaş**, Zeki Coşkuner*, Sebahattin Devecioğlu*

* Physical Education and Sports School Firat University, Elazı̆̆, Türkiye

** Physical Education and Sports Teacher

\section{Introduction}

Human body is to show an adaptation to the regular exercise, as physical and physiological. This adaptation needs specific performance and ability as a result of the special exercisers which consist of loading, duration and frequency principles. Football is seen that these principles will reveal the best way to give direction to our work.

Football has aerobic and anaerobic characteristics comprising such factors speed, strength, agility, flexibility, balance, suppleness, muscular endurance and coordination.

\section{Methods}

This research was carried out in order to search impacts of quick strength and aerobic endurance trainings on male amateur footballer's blood lipid metabolism (Cholesterol, Triglyceride, Glucose, Ldl, Hdl, Vldl), on totally forty footballers (18-28 ages) in Diyarbakır city first amateur football league.

Before the new season (Season of 2008-2009), first measurements of footballers were done by taking their 
bloods for pre-training. Then quick strength training was applied a day in a week and other a day aerobics endurance training was applied to 40 footballers with regular football training for 5 months.

At the end of regular training for 5 months, post-training second measurements were done by taking their blood samples.

By comparing the blood samples with pre-training and post-training we investigated the impacts of quick strength and aerobics endurance training on male amateur footballer's blood lipid profile.

\section{Results and discussion}

SPSS 15.0 program was used for statically analyses and its frequency, average, standard deviation ranges interpreted by helping tables. In addition, binary comparisons was made by using matched student's t test in level of $p<0.01$.

\section{Conclusion}

We found the mean age of footballers $20.88 \pm 2.68$ years, the mean age of sport $8.23 \pm 2.16$ years, and the mean height of $175.40 \pm 0.55 \mathrm{~cm}$. Also the mean weight of footballers was $72.73 \pm 3.86 \mathrm{~kg}$ in pre-exercise and $72.41 \pm$ $3.89 \mathrm{~kg}$ in post-exercise. The average difference is $0.32 \mathrm{~kg}$. respectively.

We may conclude that quick strength and aerobics endurance trainings for 20 weeks change footballer's blood lipid profiles and body compositions positively.

\section{Influence of AR-based intervention in therapy of patients with movement disorders \\ Lutz Schega, Daniel Hamacher \\ Faculty of Medicine, Department of Occupational Medicine at Otto-von-Guericke-University, Magdeburg, Germany}

\section{Introduction}

In focusing on the technological paradigm of AmI (Ambient Intelligence) we have postulated a new approach in Rehabilitation. The aim of our scientific efforts is oriented to implement an approach on computerised network of information in order to enhance our daily life in case of movement restrictions or disorders. Therefore we aim for to develop a diagnostic and intervention tool applicable in patients with different movement restrictions or disorders. This approach is necessary, especially due to the increasing count of movement problems of old and older people in our community.

\section{Methods}

In order to affect distinctions of movement restrictions or disorders of women aged between 50 to 65 years with and without hip endoprosthesis we used four methodological approaches: First we used the Heart Rate Variability (HRV, RS $800 \mathrm{CX}$, Polar) to describe the psycho-physiological adaptation. Second we utilized the electromyography
(EMG, 16-channal Telemetry, Noraxon) to characterise relevant muscle activities for hip movement. Third we applied a full-body inertial motion capture system (MOVEN) for clinical gait analysis. The fourth approach was the so called SDA-M-method (Schack 2004) as new experimental method to gain information about the cognitive structure in long term memory.

\section{Results and discussion}

The HRV showed no differences during gait analysis. Based on biomechanical and neuro-physiological gait analysis we could observe some disturbances of movement behaviour. Muscle activity pattern have indicated definite distinctions of both healthy women and women with hip endoprosthesis. Information about the restricted locomotor system by the mental representation of the movements of our women confirms findings by Schack et al. (2010) in that target group.

\section{Conclusion}

We may conclude that based on this interdisciplinary approach we gain valuable information about movement behaviour from different points of view. In fact on the one hand that approach opens a new scientific perspective to estimate gait restrictions and disorders and on the other hand based on that diagnostic and intervention tool we might be able to enhance the rehabilitation process.

\section{Literature:}

Schack T. (2004). The cognitive architecture of complex movement. International Journal of Sport and Exercise Psychology, 2(4), pp. 403-438.

Schack T., Engel, F. \& Heinen T. (2010, in press). Motor rehabilitation in walking - age dependent differences in cognitive structure between adults and seniors. European Journal of Sport Science.

\section{Trajectories of the physical activity level for elderly people during a year follow-up \\ Philipe de Souto Barreto, Anne-Marie Ferrandez \\ UMR 6578 Laboratory of Anthropologie Bioculturelle, Université de la Méditerranée, CNRS, EFS, Marseille, France}

\section{Introduction}

Knowing the aspects related to changes on the level of physical activity is an important step to promote a physically active lifestyle. The purpose of this work was to identify aspects able to determine changes on the level of physical activity in an elderly population during a year follow-up.

\section{Methods}

Two hundred twenty-five individuals $(37.3 \% \mathrm{men})$, age 60 years or over (mean $=69.5$ year, \pm 7.3 ), completed a self- 
reported questionnaire on physical activity, functional limitations, and general health. Physical activity level was rated as "low", "moderate", or "high". A dichotomous variable was created by grouping together "high" and "moderate" into "active" versus "inactive" ("low" level). Four types of trajectories of physical activity level were found. They formed four groups as follows: (1) Active-Active ("A". Persons who were active at baseline and 1 year later); (2) Inactive-Active ("IA". Those who were inactive at baseline, but active after 1 year); (3) Active-Inactive ("AI". Individuals who were active at baseline, but inactive 1 year later); and (4) InactiveInactive ("I". Those who were inactive at baseline and 1 year later).

\section{Results and Discussion}

Groups did not differ regarding sex or co-habiting in couple. ANOVA and post-hoc tests showed that persons in group "A" $(n=148)$ and "IA" $(n=20)$ were younger and better satisfied with their body functioning than those in group "I" $(n=31)$; group "A" also had less chronic diseases than group "I". Functional limitations also differed among groups. A multiple logistic regression model showed that functional limitation was related to trajectories of physical activity, even when the model was controlled for age, sex, co-habiting in couple, and self-reported health; the worst the functional limitation, the lower the probability of belonging to group "A", "IA", or "AI" $(n=26)$ rather than to the "I" group. Unexpectedly, men had a lower likelihood to be ranged in group "A" rather than in group "I". Moreover, the higher the age, the lower the probability to belong to group "A" rather than to group "I". People who did not co-habit in couple had a higher probability to belong to group "AI" rather than to group "I".

\section{Conclusion}

Sex, age, and co-habiting in couple are important factors for determining physical activity trajectories. Furthermore, trajectories of physical activity level are determined, at least partially, by functional limitations in the elderly. Improving or maintaining good levels of functional limitations is probably a way to promote a physically active lifestyle among older adults.

\section{Effects of moderate physical effort on postural stability among elderly men}

Stemplewski Rafal, Maciaszek Janusz, Osinski Wieslaw, Szeklicki Robert, Salamon Artur

Department of Theory of Physical Education and Anthropomotoric, University School of Physical Education in Poznań, Poland

\section{Introduction}

The aim of the study was to estimate differences between pre and post moderate physical effort values of posturo- graphic parameters in static and dynamic condition among elderly men.

\section{Methods}

28 elderly men (71.6 \pm 4.9 years, range $65-81$ years) participated in the study.

Postural stability were measured with use of AccuGait System posturographic plane (AMTI PJB-101 model, AMTI, Waterdown, MA). Measurements were done before and immediately after physical effort, in two options:

1) postural sway (PS) during quiet stance (parameters: range of sway in anterior-posterior AP and medio-lateral ML directions, mean radius, average velocity and elliptic area of COP sway),

2) approximate values of limits of stability (LOS-COP) during whole body leaning forward, backward and sidewards (parameters: range of whole COP displacement in AP and ML directions and calculated area of COP displacements).

Physical effort lasted $10 \mathrm{~min}$ and was performed on cycloergometer Lode Excalibur Sport (Lode B.V. Groningen, the Netherlands). Level of intensity of effort was estabished with use of Karvonen equation (level of heart rate-HR with assumed intensity level of $60 \%$ VO2max). Values of HR before effort were significantly lower than during effort $(p \leq 0.001)$ and it amounted to $72.1 \pm 8.0 \mathrm{bpm}$ i $112.3 \pm 10.7 \mathrm{bpm}$, respectively. Mean load during effort amounted to $95.3 \pm 16.7 \mathrm{~W}$ and $1.2 \pm 0.2 \mathrm{~W} / \mathrm{kg}$ of body mass.

Wilcoxon signed-rank test was used to compare values of individual parameters of PS and LOS-COP measurements before and immediately after effort.

\section{Results and discussion}

Comparison of mean values of posturographic parameters before and immediately after effort showed significant increase of average velocity of COP sway in PS measurement $(p \leq 0.001)$. The other parameters both in PS and in LOS-COP measurements didn't differ significantly. Obtained results indicated slight effect of applied effort on postural stability level. However wide range of individual reaction was observed.

\section{Conclusion}

The lack of significant differences in case of most parameters is an effect of averaging of wide ranges of individual changes which could be connected to different functional level of subjects, tolerance of physical effort, level of physical activity and many other factors.

However observed difference in average velocity of COP sway may be important taking into account that this parameter is consider as one of the most significant indices of fall risk among elderly people (Piirtola, Era 2006).

\section{References}

Piirtola M., Era P. (2006). Force platform measurements as predictors of falls among older people - a review. Gerontology 52: $1-16$ 
Aerobic Exercise and Multivitamin Supplement Alter TNF- $\alpha$ as Risk Factor of Cardiovascular Disease in Obese Women

Bakhtyar Tartibian ${ }^{1}$, Zahra gharehbagh ${ }^{2}$ \& Behzad Hajizadeh $^{2}$

${ }^{I}$ Department of Physical Education and sport science., Faculty of Humanities and Literature, Urmia University, Orumieh., Iran

${ }^{2}$ Department of Physical Education and sport science. Faculty of Humanities and Literature, Urmia University, Orumieh., Iran

Introduction: Increased level of tumor necrosis factor- $\alpha$ $(\mathrm{TNF}-\alpha)$ serve as risk factor cardiovascular disease. We examined whether the production of TNF- $\alpha$, is influenced by multivitamin supplement during aerobic exercises program.

Methods: Obese sedentary women (25-50 yrs. and BMI> $30 \mathrm{~kg} / \mathrm{m}^{2}$ ) were randomly assigned to one of the ExerciseSupplement $(n=10)$, Placebo $(n=10)$, and Control $(n=10)$ groups. The Exercise-Supplement and Placebo groups completed 9 weeks, 3 days per week aerobic exercises program with the intensity of $60-65 \%$ of predicted maximal heart rates. The Exercise-Supplement and Placebo groups received either multivitamin capsule or placebo capsule per day, for 63 days before aerobic exercises. Plasma levels of TNF- $\alpha$ were measured pre and after the end of 9 weeks aerobic exercises program and were assessed using commercially available ELISA-kits.

Results and discussion: The results show the aerobic exercises significantly decreased the level of TNF- $\alpha$ in the Exercise-Supplement and Placebo groups in the comparison with the baseline $(P=0 / 006$ and $P=0 / 004)$ conditions and control group $(P=0 / 001$ and $P=0 / 025)$ respectively. There were no significant difference between two experimental groups $(P=0 / 838)$.

Conclusion: This study suggests that multivitamin supplement and aerobic exercises with the moderate intensity have potential anti-inflammatory properties and may decrease incidence of cardiovascular diseases in obese women.

Key word: Aerobic exercise, multivitamin, pre- inflammatory cytokine, obese women

Relationship between global cognitive status, functional balance and risk of falls

Camila Vieira Ligo Teixeira, Lílian Teresa Bucken Gobbi, Jessica Rodrigues Pereira, Deysi Terumi Ueno, Thays Martins Vital, Salma S. Soleman Hernández, Luciana Mendonça Arantes, Sebastião Gobbi

Instituto de Biociências, UNESP - Univ. Estadual Paulista, Laboratórios de Atividade Física e Envelhecimento (LAFE) de Estudos da Postura e Locomoção (LEPLO), Rio Claro SP, Brazil

Introduction: The incidence and prevalence of falls in the elderly can also be due to some features of the aging process or factors associated to it, such as cognitive and motor declines. Cognitive decline and dysfunction of balance may be related and lead to falls and loss of independence. The aim of this study was to analyze a possible relationship between cognitive functions and functional balance.

Methods: Seventy-nine community-dwelling older people were evaluated by means of The Mini Mental State Exam (MMSE) aiming to assess cognitive functions, and the Berg Balance Scale (BBS), for balance and risk of falls assessment. Spearman's test was used to calculate the correlation between the variables. The significant level chosen was $5 \%$.

Results and discussion: Spearman's coefficient showed a significant $(p<0,03)$ correlation $(\mathrm{rs}=0.26)$ between MMSE and BBS scores. It cannot be denied that it is a weak correlation, numerically speaking. However if we bare in mind that cognitive functions, functional balance and risk of falls depend on multiple factors, the correlation found seems to represent an important finding. Although correlation analyses cannot be taken to explain any causal effects, it is possible that by implementing interventions such as exercise program, improvement on one of the variables may have a positive impact on the other and vice-versa.

Conclusion: The interpretation of the results allows us to conclude that cognitive functions and functional balance are related.

Acknowledgements: CNPq; FUNDUNESP, PROEX.

The study of effect of eight weeks of elected aerobic work on muasure of density of fatness of livers cells of sicks of having of fat liver (steatuhepatit)o f Shooshtar town Valizadeh $\mathrm{R}^{1}$, Nikbakht $\mathrm{M}^{2}$, Davodi $\mathrm{M}^{3}$, Asle aseidipor $\mathrm{S}^{3}$ ${ }^{1}$ Islamic Azad University, Omidieh Branch

${ }^{2}$ shahid chamran University

${ }^{3}$ Islamic Azad University, Shooshtar Branch

\section{Introduction}

The disease of fit liver is fitness gathering (more of kind triglyceride)

In liver cells (hepatosit) that providing of lock of control it will have the garlic of large virulent to destroy liver fiber and cell destruction. so the purpose of present study is the study of effect of 8 weeks elected aerobic works on measure of density of fatness of liver cells. 


\section{Methods}

This research is application and its plan to tentative and experimental with present and after test in two groups of tentative and control are done. in done present study from sonography Was used as indicator of distinction assessment of test.

\section{Results and discussion}

The founds of results of comparison between two groups of experimental and control shows that 8 weeks of aerobic work $a<0.05$ and meaningful surface ( $p=$ 0.001 ) like meaningful was caused the decrease of measure of density of fatness of liver cells of experimental group.

\section{Discussion}

With due attention to this that the disease of fit liver has important relation with metabolic dangers that fatness is one of the metabolic factors too.

The decrease of body weight and following that the decrease of fats of around liver and blood fat is that including to cholesterol and triglyceride too, can be a hope for cure of disease of fat liver that in present research, done aerobic work by experimental group could follow this goal. On the one hand, obtained results of this research coordinate with the results of many researches $(1,2)$..

\section{Conclusion}

Since, regular exercise work lead to be the increase energy use and the improvement of oxidation of skeletal fats and also the decrease of fiber and ventral fat masses so, to the sicks of having fat liver recommend that for decrease the fats of sides of self liver follow aerobic works.

\section{References}

1-Aiger, E., Datz, Ch.(2008).Iran Perturbation In Human Non-Alcholic Fatty Liver Disease . Department Internal MedicineSalzburg,Austria.8(3) 213-220.

2-piano,D, Wagner, L .(2007).Metabolic \& Nutritional Profile of Obese Adolescent With Non Alcholic Fatty Liver Disease . Journal of Pediatric Gastroentrology \& Nutrition ., 44(4) 446452

The intensity of physical exercise is an influential factor in the improvement of mobility function in elderly women with cognitive impairment

Varela S, Ayán C, Cancela JM

Faculty of Education and Sport Science. University of Vigo

\section{Introduction}

Physical deterioration in healthy elderly people is provoked by the ageing process, but this natural decay is more accentuated in individuals with cognitive deterioration, which increases the risk of injuries, falls or bone fractures among this group of population. The beneficial effects of physical activity on elderly people are widely documented but an adequate action protocol to be followed has not so far been established. The aim of this study was to analyze if the intensity of physical exercise is an influential factor in the improvement of mobility function in elderly women with cognitive deterioration. Timed Up and Go (TUG) test was chosen for the purpose because there is a close relation between the time employed to perform the test and the degree of mobility function in elderly people.

\section{Methods}

Twenty elderly women (Aged $=80,9 \pm 6,9$ years) with cognitive deterioration (MMSE $=19,3 \pm 4,6$ ) who reside in a geriatric care institution and were functionally dependent participated voluntarily in the present study. The physical activity program consisted of $20 \mathrm{~min}$ of pedalling in horizontal bicycle at an intensity of $40 \%$ or $60 \%$ of maximum heart rate (MHR) 3 times a week for a period of 3 months. The control group carried out a memory workshop instead of physical activity. After the 3 months of the intervention, physical activity ceased as well as the memory workshop. A follow-up study of the sample was carried out during the 3 subsequent months. TUG(s) measurements were taken from the 3 groups at 3 different moments: before starting the activity, at the end of the intervention stage and at the end of the follow-up study.

\section{Results and discussion}

No significant differences were observed among the 3 groups, although some improvement was noticed in the TUG scores obtained by those groups that performed physical activity compared to the control group (a reduction of $2.57 \mathrm{~s}$ between the first and last measurements in the $40 \%$ MHR group, a reduction of 3.60 in the $60 \%$ MHR group and an increase of $0.27 \mathrm{~s}$ in the control group). The comparison of the activity groups reveals a more accentuated improvement in the $60 \%$ MHR group than in the $40 \%$ MHR group (60\% MHR group was 1.03 better between the first and last measurements). It is also worth pointing out that the $40 \%$ MHR group maintained better the achieved improvement than the $60 \%$ MHR group, which lost some of it during the follow-up study stage.

\section{Conclusion}

Aerobic physical activity improves mobility function in elderly women with cognitive deterioration and if that activity is performed at moderate intensity it can be expected a more accentuated improvement than at lower intensity.

\section{Coordinative Nordic Walking as a programme in fall prevention}

Wollesen, B., Piper, J., Nagel, V., Mattes, K

University of Hamburg, Germany 


\section{Introduction}

Exercises including muscle strength and balance training can improve balance performance and prevent falls in elderly (Gillespie et al., 2005). Previous work resulted in positive effects on fall risk factors (especially balance and cardiovascular regulation) for Balance \& Coordination training (B\&C) and Nordic Walking (NW) (Wollesen et al., 2009). In the present study we combined these two training programmes to a Coordinative Nordic Walking programme (CNW) to examine whether the new intervention can provide fall prevention as well.

\section{Methods}

Using a randomised procedure, seniors $(N=90, \varnothing 65 \mathrm{Y} / \mathrm{A})$ were divided into three groups: $\mathrm{B} \& \mathrm{C}(n=31), \mathrm{NW}(n=30)$ and CNW $(n=29)$. Each group received standardised training (over 12 weeks, once per week, $60 \mathrm{~min}$, heart rate at $128-135 \mathrm{bpm})$. One-leg balance during a $45 \mathrm{~s}$ trial was recorded using an AS200 (LUKOtronic) video camera system (pre-post-design). The total balance time (TBT) of the one-leg stance was generated. The RMS of the head was calculated for motion along $\mathrm{x}-, \mathrm{y}-$, and $\mathrm{z}$-axis. The collected data were analysed by using a 3-way ANOVA (group, RMS left/right, repeated measurement; Statistica) within common limits of significance $(\leq 0,05)$.

\section{Results and Discussion}

All programmes improved balance which means that more participants were able to manage the $45 \mathrm{~s}$ one-leg stance. Only B\&C increased TBT from 42,1 s (t1) to 44,1 s (t2) $(p=0,03)$. These findings suggest that $\mathrm{B} \& \mathrm{C}$ is more effective to improve balance even for those elderly who were not able to complete the $45 \mathrm{~s}$ one-leg trial. RMS for head motion (right leg) showed an increase of $5,5 \mathrm{~mm}$ in $\mathrm{B} \& \mathrm{C}$, but a decrease of 2,4 $\mathrm{mm}(\mathrm{NW})$ and 1,5 $\mathrm{mm}(\mathrm{CNW})$ in the other groups $(p=0,07)$. According to Meyer \& Ayalon (2006) and Lord (1995) reduced postural sway means improved balance performance and reduced fall risk for seniors. However our findings suggest that the discussion to decrease postural sway to improve balance should be broadened even further and include the concept of an active functional postural sway as a method to conceive successful balancing exercises.

1. Gillespie, L. D., Gillespie, W. J., Robertson, M. C., Lamb, S. E., Cumming, R. G. \& Rowe, B. H. (2005). Intervention for prevention falls in elderly people. Cochrance review, 4 .

2. Wollesen, B., Nagel, V. \& Mattes, K. (2009). Balance Training vs. Nordic Walking-Effects on Balance Performance of Seniors, $11^{\text {th }}$ International Conference of Sport Kinetics, 27, 88f.

3. Meyer, G. \& Ayalon, M. (2006). Biomechanical aspects of dynamic stability. Eur Rev Aging Phys Act, 3, 29-33.

4. Lord, S. R., Ward, J. A., Williams, P. \& Strudwick, M. (1995). The effect of a 12-month exercise trial on balance, strength, and falls in older women: a randomized controlled trial. JAGS, 43; 1146-54.

\section{Physical activity programs}

Law regulation and government policy in georgia in the sphere of culture and sports with regard to people of advanced age

George Chiladze, Darejan Kobelashvili

Ilia State University, Tbilisi, Republic of Georgia

By Georgian legislation, any advance age person has right of any kind of creative (intellectual, cultural, sporting) activity according to his/her interests and abilities. Freedom and independence of creative activity is guaranteed. It is not allowed to interfere in creative process, establish a censorship on it, impose a seizure on piece of creative work and forbid its distribution, except those occasions when it infringes upon other person's rights, legal interests, stirs up and provokes nationalistic, ethnic, religious and racial animosity, propagates war and violence, promotes pornography, etc.

The right of the personal cultural originality and sport activity is guaranteed. Each advanced age person's right of protection of his originality, realization of his activities and free selection of his aesthetic orientation. Each citizen of Georgia is obliged to respect and observe the principles and norms acknowledged in the sphere of sports and culture. The legislation recognizes the priority of the right of a person engaged in creative work. Rights connected with cultural and sport activity of a creative person have priority over the right of other subjects of cultural and sport activity — state and legal entities.

Realization of rights of a private person are exercised on the base of the Georgian legislation and the international duties taken by the state. According to Constitution, each person has right of receiving unrestricted art and sports education. Financing of the basic art education is ensured by state. The right of proprietorship in the area of culture and sport activity is guaranteed.

Mutual responsibility on protecting the sports values between citizens creates the appropriate conditions for social relations, economics, law-abidance and conscientiousness, however these relations represent the relations under private law.

Mission and goals of the government of Georgia in the sphere of education, culture and sports with regard to the advanced age people are outlined in the Government program and provide for addressing $1 / 3$ of the budget will be directed towards the social programs, increase of employment by way of active economic activity, substantial reduction of poverty, completion of pension reform and increase of the volume and quality of the pension package, development of healthcare 
infrastructure, accessibility of medical care in rural areas, development of public/compulsory insurance police, continuation of the praiseworthy beginning program, etc.

Especially is worth mentioning the Program on rehabilitation of the sports infrastructure that is to be implemented which provides for construction of about 50 sports centers all across Georgia which will be compliant to the modern standards. Certain portion of the program is also aimed at development of sports and rehabilitation centers were people of advanced age will have possibility to visit and strengthen their health and physical condition.

\section{References}

1. George Chiladze. Legal Influence on the Culturological Relationships and Legal Regulation

Mechanism in the Sphere of Culture. The International Caucasological Research Institute's

Journal AMIRANI, N 17-18, Montreal - Tbilisi, 2007, pp. I60-171.

2. George Chiladze. Some Aspects of Legal Duty in the Sphere of Culture. The Illternational

Caucasological Research Institute's Journal AMIRANI, N 17-18, Montreal - Tbilisi, 2007, pp.172-187.

3. George Chiladze. Olympic Pedagogy, Olympic Culture and Intellectual Property in Georgia

- Culturological and Economical-Juridical Aspects. The $2^{\text {nd }}$ International Symposium on

Olympic and Paralympic Pedagogy. Tehran, 2008, 23-24

February.

4. Georgian Law "Concerning Culture”. Tbilisi, 1997

5. Georgian Law "Concerning Sport". Tbilisi, 1996

6. Program of the Government of Georgia. Tbilisi, 2008

\section{Therapists to deliver a home-based exercise programme to elderly patients}

Timo Hinrichs ${ }^{1}$, Claudio Bucch ${ }^{1}$, Anna Moschny ${ }^{1}$, Michael Brach $^{2}$, Juliane Volke ${ }^{1}$, Ulrike Trampisch ${ }^{1}$, Stefan Wilm ${ }^{3}$, Petra Platen ${ }^{1}$

${ }^{I}$ Department of Sports Medicine and Sports Nutrition, University of Bochum, Germany

${ }^{2}$ Institute of Sport Science, University of Münster, Germany

${ }^{3}$ Institute of General Practice and Family Medicine, University of Witten/Herdecke, Germany

\section{INTRODUCTION}

Exercise helps to preserve functional status and mobility in old age. Sedentary, chronically ill or frail elderly people, however, are difficult to reach for interventions if they live in their own homes. A home-based exercise programme, approaching and supporting those elderly via their general practitioner (GP) and an exercise therapist, is topic of research with regard to feasibility (Trial registration: ISRCTN58562962).

\section{METHODS}

The study was designed as a single arm interventional trial. Participants were recruited through their GPs. The 12-week intervention consisted of physical activity counselling, a home-based exercise programme (home exercises to improve strength, flexibility and balance, and walking for exercise to improve aerobic capacity), and consultations provided by an exercise therapist in the GP's office and via telephone. Adverse events were documented and judged by the GP. At the end of the intervention, participating GPs were asked if they would participate in the trial again.

\section{RESULTS}

Eight GPs from a convenient sample in the state of Northrhine-Westfalia participated in the trial. A final number of 91 patients (median age 76 (range 70-87) years; $60 \%$ females) completed the baseline assessment and started the intervention in 2009. According to their GPs, $85 \%$ of participants had been diagnosed with arterial hypertension, $32 \%$ with diabetes, $32 \%$ with coronary heart disease, $20 \%$ with chronic heart failure, $15 \%$ with chronic obstructive lung disease, $66 \%$ with degenerative spine disease, $28 \%$ with knee osteoarthritis, $21 \%$ with hip osteoarthritis, and $14 \%$ with osteoporosis. Seventy-six participants (84\%) completed the intervention and attended the final assessment. Eighteen adverse events (in 16 participants $(18 \%))$ were documented by the GPs. Ten participants (11\%) had to abort the intervention due to an adverse event. According to the GPs' judgement, no adverse event was caused by exercise. Further reasons for aborting the intervention were: time constraints ( 3 participants) and fear of negative health outcomes ( 2 participants). All GPs stated that they would participate in the trial again.

\section{CONCLUSION}

A new kind of cooperation between GPs and exercise therapists to deliver a home-based exercise programme has demonstrated good feasibility. To evaluate the effects, a randomized controlled trial should be conducted.

The study was conducted within the research cooperation PRISCUS and funded by the German Federal Ministry of Education and Research (01ET0720).

\section{Effects of different exercise interventions on cognitive functioning in older adults \\ Illig Cathleen \\ University of Leipzig, Department of Exercise and Sport Psychology, Leipzig, Germany}

\section{Introduction:}

Aging is associated with decline in cognitive performance. In a meta-analysis of RCT-studies, Colcombe and Kramer (2003) are able to show convincing evidence for effects of exercise on cognitive functions. According to their results, 
aerobic exercise and resistance training have moderate effects on cognitive functions. Angevaren et al. (2008) support the evidence for effects of aerobic training and LiuAmbrose and Donaldson (2009) also find positive effects of resistance training. But Colcombe and Kramer (2003) also find that a combined aerobic and resistance training is the most effective one. Actually is unclear which kind of exercise is the most effective one. The study compares two three month intervention programs (resistance training (EGr) and combined aerobic and resistance training (EGc)) against a gymnastic control group (CG) on their effects of the cognitive functioning.

\section{Method:}

In a randomized controlled trial two points of measurement were conducted: one directly before (T1) and one at the end of the intervention (T2). The sample consisted of healthy adults $60+$. At T1 $N=59$ participants could be tested (EGr $n=22$, EGc $n=18$, CG $n=19,34$ female; age $=$ 68 years, $S D=4.9$; range: $61-88$ ). At T2 47 participants (EGr $n=20$, EGc $n=14$, CG $n=13$ ) could be tested again. The EG's trained $60 \mathrm{~min}, 3$ times a week. The CG $60 \mathrm{~min}$ once a week. To measure cognitive functions different standardized tests (attention, memory, working memory, word fluency) were used as dependent variables. Using an ANCOVA with contrasts the effects of the intervention have been analyzed.

\section{Results:}

Results show that there were no effects of the interventions on attention, memory and working memory. But there was a significant increase of the combined training group in word fluency whereas the resistance training group could not increase significantly.

\section{Discussion:}

The study shows a significant effect of the combined aerobic and resistance training on word fluency. So a combined training is the most effective one to enhance cognitive functions. Actually there is a second wave to enhance the sample size. Due to the increase in power it may be possible to find effects of the interventions on the other cognitive functions too.

\section{References:}

Angevaren, M., Aufdemkampe, G., Verharr, HJJ, Aleman, A. \& Vanhees, L. (2008). Physical activity and enhanced fitness to improve cognitive function in older people without known cognitive impairment. Cochrane Database of Systematic Reviews 2008, Issue 3. Art. No.: CD005381. doi:10.1002/14651858. CD005381.pub3.

Colcombe, St. \& Kramer, A. (2003). Fitness effects on the cognitive function of older adults: a meta- analytic study. Psychological Science, 14, 125-130.

Liu-Ambrose, T. \& Donaldson, M. (2009). "Exercise and cognition in older adults: is there a role for resistance training programs?" Br. J. Sports Med., 43, 25-27
Effects of a six-month intervention program for older adults

1 Magdolna Vécseyné Kovách, 2 Judit Kopkáné Plachy,

3 József Bognár

${ }^{1}$ Eszterházy Károly College-Institute of Physical Education and Sport Sciences, Eger

${ }^{2,3}$ Semmelweis University Faculty of Physical Education and Sport Sciences, Budapest

\section{Introduction}

It is of cruisal importance in our ageing society to maintain physical fitness, quality of life and independence of the old. Regular physical activity is one of the best self-care behaviours that can contribute to a healthy ageing process. Health risk for older people of sedentary behaviour is widely researched (Evans, W. J., 1999, Keysor and Jette, 2001). Older people who have been inactive and sedentary in the past can develop a surprising degree of fitness with properly designed graduated exercise. Our recent study's purpose was to design and implement a 6 month intervention program for the elderly to increase physical activity, develop their functional fitness and psychological wellbeing. It was also aimed to compare the effectiveness of two types of exercises-Aqua-fitness and Pilates-on the above mentioned variables.

\section{Methods}

The participants were recruited from different clubs for the retired in Eger, Hungary. 55 people took part in the program, devided into three groups. The first group $(N=22, M=66,41)$, did Pilates three times per week for $60 \mathrm{~min}$, the second $(N=$ $18, M=68,17)$ did Aqua-fitness 2 times weekly and the third one was the control group $(N=15, M=64)$.

The Fullerton Functional Fitness Test was chosen to measure physical fitness: aerobic capacity, flexibility, strength, dynamic balance. Rathus Assertiveness Schedule was used to measure the changes in assertiveness and social abilities. Differences between the groups were analyzed with Anova with repeated measures in SPSS.

\section{Results and discussion}

Physical performance: there were signifiacant differences in five out of six variables in the Fullerton test in the Pilates group. Lower body strength, upper body strength, aerobic endurance, dynamic balance and lower body flexibility improved. Shoulder flexibility improved, but not significantly. All six variables improved significantly in the Aquafitness group. The Post-hoc analyses did not show difference between the exercising groups.

There was no significant difference between the pre-and post measurements in assertiveness and social abilities in neither of the groups.

\section{Conclusion}

Both Pilates workouts three times per week, both Aquafitness done regularly for a half year, appropriate means to 
improve physical performance needed for everyday life for the old, but was not enough to make any changes in their social abilities and assertiveness.

\section{References:}

William J Evans, Exercise training guidelines for the elderly. Medicine \& Science in Sports \& Exercise 31(1), Jan 1999, 12-17

Keysor, J. J. and Jette A. M. (2001). Have we oversold the benefit of late-life exercise? Journal of Gerontology: Medical Sciences. 56A, M412-M423.

Who receives general practitioner advice on physical activity? Analyses in a cohort of elderly primary health care patients (getABI)

Anna Moschny ${ }^{1}$, Timo Hinrichs ${ }^{1}$, Renate Klaaßen-Mielke ${ }^{2}$, Ulrike Trampisch ${ }^{1}$, Petra Platen ${ }^{1}$

${ }^{1}$ Department of Sports Medicine and Sports Nutrition, Ruhr-University Bochum, Germany

2 Department of Medical Informatics, Biometry and Epidemiology, Ruhr-University Bochum

\section{Introduction}

Despite all known benefits of regular physical activity (PA) on functional status, mobility and thus independence in old age, the PA level of most elderly people is insufficient compared to current guidelines. An ideal source of assistance or support for older people to become more active might be the general practitioner (GP). In their practice, GPs have regular contact to numerous elderly people, and often have long-lasting and trustful relationships with them.

Currently, data concerning GP advice on PA in Germany is missing. Therefore, the aim was to evaluate the prevalence and characteristics of elderly patients who received PA advice from their GP.

\section{Methods}

In a monitored prospective cohort study (German epidemiological trial on ankle brachial index, getABI), 6880 unselected patients aged 65 years and above were followed up by 344 representative primary care physicians in Germany since October 2001. As part of the 7-year follow-up telephone interviews, 1937 patients (median age 77; 72-93 years; $53.3 \%$ women) were asked whether their GP had given them advice to be regularly physically active, within the past 12 months. Patient characteristics were assessed on the basis of socio-demographic, medical, and activity-related parameters. Analyses comprised only complete cases with regard to the analysed variables. Statistical significance level for the multivariate logistic regression analysis was set at $p<0.05$.

\section{Results and discussion}

Out of 1458 analysed patients (median age 77; 72-93 years; $50.9 \%$ women), $32.7 \%$ stated that they had received advice to be regularly physically active. Those significantly more likely to receive GP advice on PA were men, patients more often visiting their GP, those with lower health-related quality of life, as well as those suffering from coronary heart disease and/or myocardial infarction, peripheral arterial disease, diabetes, or osteoarthritis.

\section{Conclusion}

It is well known that most elderly people are insufficiently active and would greatly benefit from increased PA. Nevertheless our results revealed a relatively low prevalence of GP counselling on PA among elderly primary care patients. Barriers to give advice on exercise to older patients might be lack of formal clinician training in PA counselling, lack of reimbursement, or time constraints. GPs seemed to focus their PA advice on patients in need.

\section{The Effect of a Selected Walking Program on Reaction Time and Knee Extension Strength in 60-75 Sedentary Aged Men \\ Alireza Rahimi, Vahideh Safaeinejad \\ Faculty Members of Islamic Azad University.Karaj Barnch}

\section{Introduction}

As we age, muscle strength declines, with most of the decline occurring after age fifty. The loss of strength is associated with the loss of muscle mass, due to a decrease in the number of both Type II fibers. The loss of Muscle fibers seems to be related to a neurological change at the level of the motor neuron, and whatever affects the fibers attached to it.(1)

\section{Methods}

The purpose of this study was the effect of a special walking program on Reaction Time and Knee Extension Strength in 60-75 sedentary aged men. In this respect, 24 aged men who for their pastime went to the parks and who already did not participate in the athletic programs and heavy body activities, voluntarily participated in this study. First, the study samples were examined by the heart specialist, and then their total health was approved for the participation in the walking program. The average age of the participants was 65 and their average weight was $76 \mathrm{~kg}$. After the pre test, the participants of this study, were randomly divided in to two experimental group $(n=12)$ and the Control group $(n=12)$. The participants of the experimental group took part in the walking exercise for 6 weeks and each week for 3 sessions, (18 sessions). And the Control group just focused on the daily and regular program. After the end of the 18 session, again, tests containing: reaction time and knee extension strength were measurement in two groups and findings analyzed.

\section{Results and Discussion}

For the analysis of findings, using of t-test for the dependent and independent groups in the significant line 
of $P \leq 0 / 05$; . The results of this study showed that; walking exercises had positive and significant effect on reaction time in sedentary aged men.Also, results indicate of the positive effects of walking exercises on the knee extension strength, but this effect was not significant statistically.

\section{Conclusion}

The findings of this study suggest that walking programs for the improvement or the reserving important factors like the reaction time in the daily programs should be placed in the aged men' lives.

\section{References:}

1-Lynn Aley et al; - Effect of age, task complexity, and Exercise on Reaction time of woman During Ambulation tasks. Pubs Journal. 2007

\section{Physical activity and ageing: professional competencies of employees working in the nursing homes and pensions for seniors \\ Julie Wittmannová \\ Faculty of Physical Culture, Palacký University, Olomouc, Czech Republic}

\section{Introduction}

This article presents some results gathered during the research project ESF No. CZ.04.1.03/3.2.15.2/0274 "Training of pedagogical Professional for leisure time activities of persons with special needs". Aim of the project was to increase the quality of education in this field by presenting the new study module. One of the goals was analyzing of professionals competencies required by employers and we are presenting results from institutions providing social services for seniors (nursing homes, pensions for elderly).

\section{Methods}

It was used two techniques for data collection. First onequestionnaire of own construction was send to 704 institutions providing social services for seniors (nursing homes, pensions for elderly). Respondents was asked to marked adequate value (from 1 to 5 -most important) for competencies divided into 6 categories (social field, communication, theoretical competencies, practical competencies, information technology field, professionals fields). Second technique was semi structured observation and interviews with responsible employers.

\section{Results and discussion}

Backflow of questionnaire was only about 20\% (127 answers). Short summary of results in separated domainsThe results show the need of preparation professionals in the social field, field of theoretical competencies, communications and field of informational technology. Less is stressed the need for practical skills (physical activities, sports) and professional expertness. This profile fits more to person who is organizing social, cultural and sport leisure time activities, but less realizing and doing practically these activities. Social workers employed in institutions are typically choosing one or two preferred activities doing with clients practically, other activities leasing to organize in cooperation with volunteers, students etc. This situation may be due to Czech reality that in the institutions offering social services to elderly is not professional position of educationalist, but position of social worker or for the field of health services physiotherapist.

\section{Conclusion}

In our conclusion we recommend as good alternative to employ professionals from the field of "leisure time pedagogy for persons with special needs", it may improve the quality of approach to organization of physical activity. The employers are interested at lest about specialization courses, workshops and seminars in the field of physical activities and sport for current social and medical staff.

We strongly suggest to our Universities working in the field of kinantropology to start with at lest separate optional subjects for students to choose and think about whole complete study modules.

\section{Physical Fitness}

\section{Functional testing in senior women \\ Václav Bunc, Miroslav Stilec \\ Faculty of PE and Sports Charles University, Prague, Czech Republic}

\section{Introduction}

A small percentage of the old and oldest who are physically fit can be tested using routine physical or functional tests, such as treadmill and bicycle ergometer tests, and routine strength, flexibility, and agility tests. However, their age does present several problems, particularly if their scores are to be compared to those of younger people. The various methods currently available to assess the effect of physical activity vary greatly in their applicability in research, intervention studies, clinical practice, and personal assessment.

\section{Methods}

An effect of physical exercise in human may be assessed by level of aerobic fitness (AF) and physical performance (PF). Dynamic work of cyclic character like running, walking, swimming and cycling requires the movement of large muscle masses and thus may reflect actual state. From these activities the walking is probably the most easily accessible, and often underestimated as a general tool for assessment of AF and/or for improvement of both AF and PF. The aim of this study was to verify the possibility to use of walking for assessment $\mathrm{AF}$, and $\mathrm{PF}$ in women seniors. 


\section{Methods}

The load based on treadmill walking was used for evaluation of fitness state in a group healthy senior women $[n=89$, age $=$ $68.3 \pm 4.7$ years, mass $=69.1 \pm 7.2 \mathrm{~kg}$, height $=161.2 \pm 2.6 \mathrm{~cm}$, percentage of body fat $(\% \mathrm{BF})=37.3 \pm 5.0 \%, \mathrm{VO}_{2 \max }$. $\mathrm{kg}^{-1}-25.4 \pm 4.1 \mathrm{ml} \cdot \mathrm{kg}^{-1} \cdot \mathrm{min}^{-1}$. After the two warm up loads about 50 and $70 \% \mathrm{VO}_{2 \max }$ (HR about 65 and $85 \%$ of $\mathrm{HR}_{\max }$ ), the load was increased by $1 \mathrm{~km} \cdot \mathrm{h}^{-1}$ by each minute till the subjective exhaustion. The $\mathrm{BC}$ was assessed by whole body BIA method with use of prediction equation for seniors and functional variables were measured in open system. In the field the fitness state was evaluated by walk on $1600 \mathrm{~m}$ distance on the flat terrain. Both results are on the same level.

\section{Results and discussion}

The standards were prepared for seniors women in age ranged from 65 to 80 years. . The norms for AF were divided into three groups. "Good" level was determined as the mean values from all data for each particular age. "Excellent" values were calculated as the mean plus SD, and "Poor" values were constructed as the mean minus SD. These standards evaluate the fitness state - body fat content, FFM, ECM and BCM and AF, PF-maximal speed of walking. The mean levels of followed parameters are the predispositions for active life style and independency of senior women and thus may contribute to their integration in society.

\section{Conclusion}

The assessment of physical fitness state and mainly of physical performance in seniors may be evaluated by treadmill and/or flat terrain walking. This data may be reflected in their independency and thus in job, self-care, household chores, home and yard maintenance, transportation, and discretionary leisure-time activities including fitness-promoting exercise and sports.

\section{Study was supported by grant of Czech Ministry of Education MSM 0021620864}

\section{The Weighted Sum: A More Accurate Way to Estimate Overall Functional Fitness}

Katerina Machacova ${ }^{1,2}$, Vaclav Bunc ${ }^{2}$, Diane Elizabeth Adamo $^{3,4}$

${ }^{1}$ ILA s.r.o., Klimentska 20, Prague, Czech Republic; ${ }^{2}$ Charles University, Faculty of Physical Education and Sports, Prague, Czech Republic; ${ }^{3}$ Institute of Gerontology, Wayne State University, Detroit, MI; ${ }^{4}$ Department of Health Care Sciences, Wayne State University, Detroit, MI

Introduction: Functional fitness is a major determinant of quality of life and one of the key predictors of many health outcomes in later life. The ability to accurately measure functional fitness is important for both research and clinical purposes because multiple evaluations are not always satisfactory or even desirable. But functional fitness is a latent construct consisting of specific components essential for independent functioning; therefore, its estimation is rather complicated. The purpose of the study was to examine the structure of the construct of functional fitness, to establish the contribution of its individual components, and based on the results suggest a novel approach to accurately estimate Overall Functional Fitness score in older adults.

Methods: The Senior Fitness Test (Rikli \& Jones, 2001), which consists of six performance-based tasks validated to measure functional fitness components such as upper- and lower-body strength, aerobic endurance, upper- and lowerbody flexibility, and agility was administered to 78 adults (mean age: $82.0 \pm 8.8$ years). The theoretical model of Overall Functional Fitness was tested using structural equation modeling performed in LISREL.

Results and discussion: The standardized maximum likelihood solution of a single level model showed satisfactory goodness-of-fit indices (RMSEA $=0.00 ; \mathrm{NFI}=0.95 ; \mathrm{GFI}=$ $0.97 ; \mathrm{RMR}=0.038$ and $\chi 2=7.64, \mathrm{df}=9$ ). Findings demonstrate that the structure of functional fitness is one-dimensional and contributions of its individual components are hierarchical in nature. Expectedly, mobility related performance (lower-body strength and flexibility, stamina, and agility) appeared to be more important than upper-body performance (upper-body strength and flexibility). Therefore, an accurate estimation of Overall Functional Fitness considers the weighted sum, rather than a simple sum, of all identified components.

Conclusion: Considering the importance of functional fitness status in later life, the ability to accurately estimate this latent construct is the first step to better understanding its role in aging processes. From the clinical point of view, using an estimation of Overall Functional Fitness contributes to implementing and evaluating effective activity/ exercise programs targeting the select needs of older individuals that become increasingly variable with the onset of diseases or disabilities.

Rikli, R.E. \& Jones, J.C. (2001). Senior Fitness Test Manual. Champain, IL: Human Kinetics.

Performance comparison of selected motor abilities in slovak elderly women

Nemcek Dagmar

Faculty of Physical Education and Sport, Comenius University, Bratislava, SLOVAKIA

\section{INTRODUCTION}

Scientific research proved indeed that physical activity is of major importance in preventing premature ageing, 
maintaining functional independence, decreasing the risk factor of primary and secondary disabilities and increasing life-long well being. Regular physical activity has powerful positive effects on both physical and psychological health. Conversely, physical inactivity has a high human cost in terms of health. It shortens the life expectancy, decreases quality of life, and limits functional independence. Appropriate level of motor abilities in elderly can make their life happier and richer but first of all can bring to them healthy life-long well-being.

\section{METHODS}

In the article we are comparing average value of achieved performance in different motor abilities in elderly women with existing norms from different age category point of view following different authors (RIKLI, JONES, 2001, NEMCEK, 2010). 129 elderly women in age from 60 to 89 years participated in our research. We divided respondents into five age categories where we provided comparison of average value of achieved performance in selected motor abilities (flexibility, balance and strength) with existing norms. For flexibility measurement we used three tests: modified sit and reach test, test of lateral spine flexion and back scratch test. For balance evaluation we used one leg stand test and 8-foot up-and-go test and measuring strength we used chair stand test.

\section{RESULTS AND DISCUSSION}

Women in three age categories $60-64,65-69$ and 70 74 years achieved average level of motor abilities in all measured test except in chair stand test, where they showed above-average level of lower limbs strength. 75-79 year old Slovak women achieved in all measured tests average level of motor abilities as well as the oldest women (8089 year old) but the difference is that in one leg stand test they showed below-average level of static balance.

\section{CONCLUSION}

Everyday physical activity also for elderly people is one of the parts of their healthy life style as well as to increase physical fitness in each age. We are not recommending providing one leg stand test for elderly who are older than 85 years of age.

\section{REFERENCES}

1. RIKLI, R.E, JONES, C.,J.: Senior fitness test manual. California State University Fullerton: Human Kinetics, 2001, $160 \mathrm{p}$.

2. NEMCEK, D.: The level of selected motor abilities in elderly women. Bratislava: Union of Recreational Physical Education and Sport, 2010, 115 p.

This research was supported by project VEGA nr. 1/ 0702/10.
The safety assessment of low-intensity resistance exercise with slow movement and tonic force generation in healthy older men

Akane Ohgane, Yuya Watanabe, Michiya Tanimoto, Naokata Ishii

National Center for Geriatrics and Gerontology, Aichi, JAPAN

\section{Introduction}

The pilot study was designed to evaluate the risks (the safety) of a low-intensity resistance training with slow movement and tonic force generation (LST) in elderly population. This regimen using a relatively low load (the $50 \%$ of 1 repetition maximum: 50\%1RM), performed slower than that in conventional low-intensity strength training and attempting to generate force as tonic as possible, has been proven to be an effective means of realizing both muscle hypertrophy and maximal strength development in young men. We compared acute responses to three different protocols in healthy older men.

\section{Methods}

Twelve healthy men ( $68 \pm 4$ yrs) performed knee extension machine exercise according to three regimens: low-intensity (50\%1RM) resistance exercise with slow movement and tonic force generation (3 s (s) for eccentric and concentric actions with no relaxing phase: LST); normal speed strategy ( $1 \mathrm{~s}$ for concentric and eccentric actions, $1 \mathrm{~s}$ for relaxing) at a high-intensity $(80 \% 1 \mathrm{RM}$ : $\mathrm{HN})$; and normal speed strategy at a low-intensity (50\% 1RM: LN). All protocols involved three sets of eight-repetitions performed and $60 \mathrm{~s}$ of interset rest period. Protocol compliance, adverse events, withdrawing number, peak systolic blood pressure (SBP) and peak heart rate (HR) during exercise were assessed. The delayed onset muscle soreness was assessed by using the visual analog scale (VAS).

\section{Results}

One aged 73 yrs having hypertension (SBP/DBP at rest: $180 / 80 \mathrm{mmHg}$ ) was enrolled after he obtained medical clearance. He participated to all the trials under taking an antihypertensive in under the controlled condition (146/64). In all men included him, protocol compliance was perfect without causing any adverse events during all testing sessions. The withdrawing number throughout all testing sessions includes LST regimen was 0 of 12 . In all types of exercises, the VAS showed a maximum value (less than $10 \mathrm{~mm}$, that was nearly "no pain") at immediately after exercise or at 1 day later and then decreased almost $0 \mathrm{~mm}$ ("no pain") at 3 days later. At any time points, no significant difference was seen between exercise regimens. The peak SBP during HN (Mean +/- SD 175.5 +/$35.5 \mathrm{mmHg}$ ) was not significantly but higher than those during LST $(150.0+/-29.0 ; P=.20)$ and LN $(148.0+/-$ $30.3 ; P=.18)$, and no difference was seen between those 
during LST and LN $(P=.91)$. The peak HR during HN $(104.2+/-13.7 \mathrm{bpm})$ was significantly higher than those during $\mathrm{LN}(91.1+/-11.5 ; P=.04)$, and no significant difference was seen between those during HN and LST $(101.0+/-22.8 ; P=.72)$ and between those during LST and LN $(P=.26)$.

\section{Conclusion}

This pilot study indicated that LST is a safe, feasible, and promising intervention for healthy older men.

\section{A comparison between elderly women from Clubs for Retired People and living a Twilight Home \\ ${ }^{1}$ Judit Kopkáné Plachy, ${ }^{2}$ Magdolna Vécseyné Kovách, ${ }^{3}$ István Barthalos, ${ }^{4}$ József Bognár \\ ${ }^{1,4}$ Semmelweis University Faculty of Physical Education and Sport Sciences, Budapest, Hungary \\ ${ }^{2}$ Eszterházy Károly College-Institute of Physical Education and Sport Sciences, Eger, Hungary \\ ${ }^{3}$ University of West Hungary, Györ, Hungary}

\section{Introduction}

Advancing age is associated with predictable sensory, motor and cognitive decreases; changes in human tissues, organ systems and functions. These age-related physiological changes have potential impact on an older person's ability to function effectively in society [1].

\section{Methods}

Women over 60 years $(N=60 ; M=77,27)$ were examined in our cross sectional study in a Hungarian city, Győr. Participants were chosen from a twilight home $(N=27$, $M=79,34$ years $)$ and clubs for retired people $(N=33, M=$ 75,57 years). The purpose of this study was that participants live in residential care facilities have lower cognitive function or some obstructive illness, lower ADL and physical fitness status than who is a member of some club and live active life. Physical fitness status was assessed by Fullerton Fitness Test (FFT) and body composition (BC) with Inbody-720 bioelectrical impedance spectroscopy. Data were analysed by SPSS 17.0 for windows with multivariate ANOVA.

\section{Results and discussion}

FFT shows significant differences in 5 measurements: lower body strength, upper body strength, aerobic endurance, shoulder flexibility, and dynamic balance. These variables were significantly better in the clubs for retired people. The lower body flexibility does not show significant differences between the two groups. The body composition of twilight home residents was significantly lower in body weight, and fat free mass. We did not find any differences in body mass index (BMI), fat mass and visceral fat mass. BMI was $(M=29,3$ and 29,6$)$ normal both in twilight home and clubs. Decreased physical fitness status of institutionalised participants was proved both by the results of FFT and anthropometry measurements. Lower body weight and quantity of fat free mass could be indicators of lower muscle mass.

\section{Conclusion}

Sedentary lifestyle has negative effect on body composition, physical fitness and independence status in an old population.

\section{References}

Salem, George J., Skinner, James S.; Chodzko-Zajko, Wojtek J.; Proctor, David N.; Fiatarone Singh, Maria A.; Minson, Christopher T.; Nigg, Claudio R. (2009): Exercise and Physical Activity for Older Adults. Medicine \& Science in Sports \& Exercise, 41:1510-1530

\section{The effect of an isotonic training program on strenght} of quadriceps muscles in aging

Alireza Rahimi, Vahideh Safaeinejad

Faculty Member of Islamic Azad University. Karaj Branch. Iran

\section{Introduction}

The good of a strength -training program is to increase the maximum amount of force that can be generated by a particular muscle group recall that isotonic exercises (includes variable-resistance exercises) involves force application with joint movement (1).

\section{Methods}

The purpose of this study was to assessment of the effect of strength training (isotonic) like Nautilus equipment (in water and on land) on quadriceps muscles in knee extension to 45 and 90 degree. 175 older men (50-60 ages) participated in this research that 60 subject selected between them and then divided to three groups: control, training on land and training in water. The subjects volunteer to participate in study. The essential purpose of this research was to assessment of the effect isotonic training on Static strength quadriceps muscle and the study about the effect isometric training was performed in the past. Muscle strength was measured by tensiometer (pre test and post test) in the 45 and 90 degree of extension of knee. exercise program included: 3 sessions in a week and for 5 continuous week (15 sessions) and three sets per-session with ten repetition (Mac Kwien).Before beginning any exercise(with Mac Kwien method)subjects performed callisthenics and stretching exercise as warm-up, and consulted with his personal physician before of test program (during tests physician presented).

\section{Results and Discussion}

The results from the t test student and ANOVA indicated: 1) Training on the land and in water had significant effect on quadriceps muscle strength extension 45 and 90 degree 
of the knee and 2) Training on the land had more effect on quadriceps muscle strength on extension of knee joint. The comparison of the water group shows a little increasing in post test phase which is not statistically significant.

\section{Conclusion}

Our finding suggest that isotonic strength training may also induce central nervous system changes, which can increase the number of motor units recruited, alter motor neuron firing rates, enhance motor unit synchronization during a particular movement pattern and result in the removal of neural inhibition. So we need more studies details about isotonic exercises its effect and its various methods.

\section{References}

1) Scott K.Powars, Edward T.Howley.Exercise Physiology. New York.2001.

\section{THENAPA II Dissemination results give a boost} in Europe for more physical activity for elderly persons with and without disabilities

Herman Van Coppenolle

Katholieke Universiteit Leuven, Faculty of Kinesiology and Rehabilitation Sciences herman.vancoppenolle@faber. kuleuven.be

\section{INTRODUCTION}

During 3 years (2004-2007) specialists in APA for elderly persons of 66 higher education institutions from 27 European countries produced educational materials to promote an active lifestyle for elderly persons: the content of a programme in APA for the elderly at master and bachelor level, a motivational DVD "Never too old to be active: The joy of movement", practical active ageing activity cards and a brochure with a European survey and recommendations.

\section{METHODS}

During the dissemination year (2008-2009) these materials were translated in 15 European languages and disseminated in all countries in institutes for higher education (physical education, physiotherapy, sports science), nursing homes for elderly persons and caregivers. In every country as many as possible elderly persons were approached, as well as the concerned ministries, sports organisations and the press.

\section{RESULTS}

A consortium was created between the universities of Amiens (France), Groningen (The Netherlands) and Verona (Italy) to start up the European Master Programme in APA for the Elderly and the motivating products disseminated the urgent message of the Thematic Network for all elderly persons with and without disabilities in Europe: "Save yourself by more physical activity".

\section{CONCLUSION}

This same approach applied in all European countries at the same time during these 4 years gave a boost to the better education of students in this matter: stimulating the responsibility and motivation of elderly persons for bringing more moderate physical activity in their daily schedule, at least $30 \mathrm{~min}$ each day. The established cooperation between the actors of the network should however be continued through the European Master in APA for the Elderly and further dissemination of the products through the website www.thenapa2.org

\section{Quality of life}

Benefit of exercise for the elderly from a health promotion programme in rural Thailand

W. Boonchalaksi, P. Muensakda

Institute for Population and Social Research, Mahidol University, Salaya, Phutthamonthon, Nakhonpathom, Thailand prwbc@mahidol.ac.th

Proper exercise can help the body maintain its good health and good looks. Good health does not occur automatically. It requires good preparation and the right form of promotion. The objectives of the study are 1) to promote the elderly in rural Thailand with good physical and mental health with proper exercise, and 2) to research and develop a health promotion programme for the elderly through collaboration with health-center staffs at sub-district level. The participatory action research was used to assess the effectiveness of community-based interventions in implementing a health promotion programme for the elderly. Two health centers at sub-district level were selected and qualitative techniques were employed. Through the use of a systematic approach and attentive evaluation, the results from the evaluation at the end of the project covered 300 elderly samples indicated that more than half of the elderly were satisfied with the health promotion activities, especially the activity on physical exercise with stick. This kind of exercise was designed by local Thai women, Aunty Boon-Mee. It comprised of 12 positions and can be easily applied with body exercise. The elderly satisfied and percieved of it. After participating in the activity, they said that it can be helped them to decrease back and hip pain. Moreover, it was also found in the study that the elderly had changed their health behaviors, they were no longer shy to exercise at home. These had never happened in the villages before. However, for the 12 positions of the exercise there are 4 main principles as follows: 1) use appropriate size and length of stick by measuring arm stretching while holding the stick, 2) should perform the 
right position of straight back, not too tight, then easily make natural movement, 3) should perform 99 times at least $10 \mathrm{~min}$, and for the beginner should start each condition with 20 times, 4) should regularly and continuously perform in order to make most effective. It should be avoid exercise position that make pain as much as possible and then simultenously add until feel comfortable. One who get diseases such as heart disease or others should consult physicians before exercise. This study try to indicate the health benefits of the proper exercise because there are many kinds of exercises. The elderly should select kind or method of exercise suitable for the physical condition and have no danger that can make good and continous exercise resulting benefit to the body. The lesson learned from the study is proper exercise would reduce the expenses to be paid for the medical treatment because good health would bring happy life which in turn resulted in the happiness of the family and the community as well.

\section{Relationship between functional tests and self-reported perception of health in elderly people}

Carvalho, Carlos; Silva, Ana; Silva, Marta; Vieira, Luisa e Martins Clarice

Research Centre for Sports Sciences, Health and Human Development (CIDESD)-Higher Education Institute of Maia (ISMAI)—Portugal

\section{Introduction}

It is well known that physical activity among the elderly is directly associated to an improved quality of life which in turn increased functional capacity and independence. Unfortunately, only a small number of elderly people do in fact, get involved in some type of regular physical activity (Chandler \& Brown, 2009). Thus, the aims of this study were 1 . to analyse the relationship between self-reported health perception and the functional capacity of the elderly; 2 . to assess the relationship between locomotion capacity and perceived health. We hypothesized that subjects who obtained better results in locomotion tests (aerobic endurance, agility/dynamic balance, lower body strength) tended to have a better self-reported perception of health.

\section{Methods}

These are preliminary results of the intervention programme developed in Maia, Portugal, involved 150 subjects and aimed at evaluating the effects of different training methodologies. However our sample was composed of 49 subjects, aged 60 to 74. The functional capacity was measured by Rikli \& Jones (1999) functional fitness battery. The self-reported health perception was evaluated by the international questionnaire "Medical Outcome Study_-36" from "Health Survey- Short Version (SF-36)". The statistical analysis was performed using
SPSS (Windows 17.0) and the level of significance maintained $p \leq 0.05$.

\section{Results and Conclusion}

The self-reported perception of health was significantly associated with functional tests $(p=.01)$. Subjects who had the greatest results in tests related to locomotion corresponded to those subjects who had the best perception of health $(p=.04)$. Therefore our sample confirmed that subjects who had a better locomotion capacity are those subjects who better classified their perception of health.

Analysis of the effects of regular walking on physical and cognitive functioning, emotional status and quality of life in middle aged and older adults

Ugur Cavlak, Orcin Atalay

School of Physical Therapy, Pamukkale University, Denizli, Turkey

Introduction: Physical inactivity is a complex and serious problem. Since mid 1990s, the importance of mild intensity (at least $30 \mathrm{~min}$.) physical activity has been recommended. Walking is one of the most recommended and popular physical activity. The aims of this study were (1) to examine the effects of regular walking on physical and cognitive functioning, emotional status and quality of life in middle aged and older adults and (2) to compare with inactive subjects.

Methods: Forty middle-aged and older adults with a mean age of $56.30 \pm 4.85$ years (range; $40-70$ ) walking for at least 1 year, at least three times a week, at least $45 \mathrm{~min}$. a day and forty inactive subjects with a mean age of $55.15 \pm$ 5.64 years (range; 40-70) were included in the study. Before testing, socio-demographics belonging to the both groups recorded. Body mass index and waist-hip ratio were calculated. Physical functioning (handgrip strength, balance performance, cardio-vascular endurance, flexibility, muscular endurance and coordination), cognitive functioning (mini mental test), emotional status (Turkish version of the Beck Depression Inventory), and health related quality of life (CDC HRQOL-4) were evaluated to compare the two groups.

Results and discussion: The results of this study showed that there were significant differences in terms of physical and cognitive functioning, emotional status and quality of life between the groups $(p<0.05)$. The regular walking group had better scores in all parameters than the inactive group. The results of this study indicate that regular walking is a safe, cheap and can easily be adapted into daily life.

Conclusion: We may conclude that regular walking improves physical and cognitive functioning, emotional status, and quality of life of middle-aged and older adults. 


\section{References:}

1. Cavlak U., Yagci N., Aslan B. U., Ekici G. (2008)-A new tool measuring heath-related quality of life (HRQOL): The effects of musculoskeletal pain in a group of older Turkish people. Archives of Gerontology and Geriatrics, Article In Press

2. Cavlak U., Telli O., Baskan E., Altug F. (2009)—An Analysis Study Describing Physical Fitness Level of Overweight Subjects. Medicina Sportiva, 17: 1046-1049

3. Asbury E. A., Chandrruangphen P., Collins P. (2006)-The importance of continued exercise participation in quality of life and psychological well-being in previously inactive postmenopausal women: a pilot study. Menopause, 13(4): 561-567

4. Castillo G., Ortega P. FB, Ruiz J. (2005)-Improvement of physical fitness as anti-aging intervention, Med Clin (Barc)., 124(4): 146-55

Notice: This study was supported by the Pamukkale University, Scientific Research Projects Coordination Unit

\section{Beyond Accessibility; Restoring Movement Mobility \\ Randy Eady, Ed.M. NCC, Ko Sha Rey Rhythms Thera- peutics, Boca Raton, FL, USA \\ Jens Freitag, Industrial Designer, Managing Partner, TEO / Industriedesign GmbH, Kassel / Germany}

\section{Introduction}

Movement, including strength, agility, balance, coordination and endurance, is a set of skills that must be continuously taught and reinforced to influence conditions such as onset of frailty. A comprehensive survey through the available research shows a high incidence of movement deficits in older populations that have a sedentary lifestyle pattern. Movement deficits refer to poor ability to utilize and control muscular systems. It may be evident in gait patterns (walking, navigating stairs), poor posture/trunk stability. This presentation features a pathfinding portable, neuro-muscular integration movement application of integrated design components that is highly accessible and supports the latest understandings of breathing, balance and bi-lateral co-ordination to improve independent mobility. Provides an overview of the on-going biomedical research protocols with these components as they influence neural repair and applications of rehabilitation strategies.

\section{Methods}

Systematic application of three protocol systems that focus on improved breathing, balance and bilateral co-ordination over a prescribed circuit for a 10-12 week period enhances symptom relief, improves recovery after neurological injury or chronic disease condition and provides caregiver respite. A system of rhythmic movement through the activity equipment is reviewed as studies have found that swimmers and rowers - both of whom practice a profoundly rhythmic form of exercise-had healthier immune systems. (1) It is presumed that movement plus rhythm tends to create an anxiety-reducing meditative state This is explained as the organic relaxation response in which bio-rhythms are reinforced when engaged in an appropriate sequence. (2)

\section{Results and Discussion}

Demonstration of easy-to-access form of recreational therapy that focuses on building core muscle strength, body symmetry and integration of the body's three organic balance centers. Program improves range of motion in walking, strength, flexibility and enhanced stability. Explains each protocol in direct correlation to the physical activity equipment featured in a specific circuit of optimum movement sequence. The breathing protocol is followed by the balance protocol and concludes with the bi-lateral co-ordination protocol. Progressive and age-appropriate process is elaborated that reverses indigenous process of coordination: starts with homo-lateral leads to bi-lateral leads to bi-lateral integration leads to independent freedom of movement in all four limbs. It is a very specilized blend of parcour (l'art du déplacement: English: the art of moving) as a physical discipline of training to overcome obstacles within one's path by adapting one's movements to the environment.

Our primary focus in Ancient Walking to Primal Rhythms is directly on developing one's level of spatial awareness to be used to aid development, mobility and integration of the body's three balance centers.

\section{Conclusion}

Rehabilitation professionals and integrative wellness practitioners that need to explore innovative, costeffective ways to narrow the gap between rehabilitation and recreation therapy and meet the unique needs of our aging population will find this approach to be highly effective.

(1) Broughton, H. R. The Journal of the Royal Society for the Promotion of Health 1987;107:131-133

(2) Benson, H. The relaxation response. Physiology, history and clinical applications. In: Adelman G, Smith BH, eds. Encyclopedia of neuroscience. 2nd edition Boston:Elsevier, 1776-8

Physical activity, falls efficacy, functional performance and quality of life in Swedish 75-year-olds Kerstin Frändin*, Helena Hörder**, Ingmar Skoog** *Department of Neurobiology, Care Sciences and Society, Division of Physiotherapy, Karolinska Institutet and **Neuropsychiatric Epidemiology Unit, Institute of Neuroscience and Physiology, Sahlgrenska Academy at Göteborg University, Sweden 


\section{Introduction}

Measures of physical activity and functional performance have been shown to be predictors of adverse health-related events in older adults, while the association to multidimensional quality of life in older adults is less known. The aim of this study was to investigate the relation between level of physical activity, degree of falls efficacy as well as functional performance and multidimensional aspects of quality of life in senior women and men.

\section{Methods}

A representative sample from the general population in Göteborg, Sweden, born in 1930, was examined at age 75 $(n=637,383$ women and 284 men, response rate $61 \%)$. Physical activity was estimated according to a six-graded scale and through interview on walking habits, while falls efficacy was determined through the Falls Efficacy Scale. Functional performance was measured as walking speed, timed chair stand, stair climbing capacity, grip strength and one leg stance. Health-related quality of life was assessed through the Short Form-36 (SF-36), which includes eight domains, and health and life satisfaction with single items.

\section{Results}

Sixty per cent of the participants walked $150 \mathrm{~min} /$ week or more. Physical activity, falls efficacy and functional performance all correlated to quality of life, but the levels varied between the different domains and the domain with the lowest association was Mental Health. Maximal walking speed was the variable that highest correlated with all subscales of SF-36, ranging from $r=0.24$ in Role Emotional to $r=0.60$ in Physical Function. After adjustment for potential confounders, a high maximal walking speed was associated with maximal score in many subscales of SF-36: Odds Ratio (95\% confidence interval) for every $0.1 \mathrm{~m} / \mathrm{s}$ increase: Role Physical; women 1.24 (1.13-1.36) men 1.16 (1.04-1.30), Vitality; women 1.29 (1.04-1.60) men 1.25 (1.02-1.54), Social Function; women 1.24 (1.13-1.36) men 1.18 (1.04-1.33).

In women, there were associations also with Bodily Pain; 1.12 (1.01-1.25), General Health 1.59 (1.15-2.20) and Role Emotional 1.17 (1.06-1.28).

\section{Conclusion}

Physical activity, falls efficacy and functional performance showed low to moderate correlations to all domains of quality of life. Maximal walking speed was the test of functional performance that strongest correlated with all measures of quality of life. After adjustment for potential confounders, maximal walking speed was associated with multidimensional aspects of healthy ageing, especially in women.

\author{
Relationship between self-rated physical activity \\ and health outcomes \\ Aivars Kaupuzs, Viesturs Larins \\ Latvian Academy of Sport Education, Riga Latvia
}

\section{INTRODUCTION}

According to the U.S. Surgeon General's Report on Physical Activity and Health, inactive people are nearly twice as likely to develop heart disease as those who are more active. Cardiovascular disease (CVD) are the largest cause of death in the EU and account for approximately $40 \%$ of deaths or 2 million deaths per year. Risk factors (smoking, high blood pressure, high blood cholesterol, overweight, and diabetes) greatly increase the chances of developing heart disease. Physical activity (PA) both directly reduces heart disease risk and enhanced health related quality of life (HRQOL) that provides "the successful aging". Outcome expectations an important element of social cognitive theory, that can predict person health behavior such as physical activity. This study examined the relationships between self-rated PA, exercise outcome expectations, heart risks assessments and HRQOL in older adults.

\section{METHODS}

For this study were recruited 44 community dwelling adults aged 60-75 years. We used interviewer-administered the International Physical Activity Questionnaire (IPAQ) short version, Multidimensional Outcome Expectations for Exercise Scale (Wójcicki 2009), JBS CVD risk index assessment and the Medical Outcomes Study short form 36-item questionnaire (SF-36).

\section{RESULTS}

The mean age \pm SD of the samples was $66.5 \pm 4.9$ years. The majority of the respondents were female $(81.7 \%)$. The results of the study shows that total physical activity is very high (mean=7798.1 MET/min/wk; SD=3626) and it is correlated with positive exercise outcome expectation ( $r=$ $0.391, p<0.01)$. The results shows high CVD risk index $(16 \% ; \mathrm{SD}=5.3)$ and $\mathrm{BMI}(29.15 ; \mathrm{SD}=4.35)$ of the samples. At this study we funded out the statistically significant relationship between PA and HRQOL questionnaire $S F-36$ data. (Pearson Correlation PA and PCS r=0.497; PA and MCS $\boldsymbol{r}=0.580 ; p<0.01)$. But gained data does not show significant correlation between PA and CVD risk index and even BMI.

\section{CONCLUSION}

Our findings approve the theoretical relationship between physical activity and HRQOL as well as with positive exercise outcome expectation. The most of the respondent IPAQ results refer to "moderate" or "high" PA level, but there is a risk of the overestimating of the total activity at this age group. That possible could explain this disagreement with many studies which data suggest that physical activity decrease CVD risk factors, but our research did not find such pathway. Although our study could not be generalized in reason of small sample, this results have provided the important background for future research in Latvian elderly population. 


\section{REFERENCES}

Wójcicki TW, White SM, McAuley E. Assessing outcome expectations in older adults: the multidimensional outcome expectations for exercise scale. J Gerontol B Psychol Sci Soc Sci., 64, 2009, 33-40.

Physical condition of older people living in residential care facilities: the direct effects of an aerobic training on motor skills and the indirect relationships with basic activity of daily living

Magistro Daniele, Mulasso Anna, Nebiolo Simona, Liubicich

Monica Emma

Motor Science Research Center, SUISM (University School of Motor \& Sport Sciences), University of Turin, Italy

\section{INTRODUCTION}

Few studies have investigated the effects of physical activity training in very old people living in residential care facilities in terms of the potential positive consequences for the general physical conditions. At very old ages, the biological potential of the individuals is even further weakened, leading to different forms of frailties which contribute to make older people much vulnerable and less apt at mastering the tasks of daily living. The present study is aimed at investigating the changes between pre-test and post-test, after having introduced an aerobic programme of physical activity (two sessions, each of $45^{\prime}$ per week for 16 weeks, over a span of roughly 4 months, delivered by specially trained instructor) in motor skills and on autonomy of Activities of Daily Living (ADLs) in a sample of elderly people.

\section{METHODS}

Thirty-one elderly $(84,59 \pm 7,26$ years), 21 women and 10 men, classified as partially or totally self-sufficient and living in two residential care facilities in northern Italy were enrolled in the study. We collected baseline and post-test measurements for Tinetti Test, hand and fingers strength, anthropometric datas (height, weight, BMI, waistline), Basic Activities of Daily Living (BADL) and Short-Form (SF)-36. The datas were analyzed with Wilcoxon test-Not parametric statistics for dependent samples, effect size (ES) and regression analysis.

\section{RESULTS AND DISCUSSION}

The preliminary results show that: a) the finger strength of right hand increases between baseline and post-test $(p<.05$; ES= $34)$; b) there is a strong and positive relationship between the finger strength of right hand and the development of vigorous activities $(p<.002 ; \beta=.568)$ at the end of motor activity period; c) exists a tendency of improvements in autonomy of some ADLs (dressing $(p=.08)$; toilet use $(p=.11)$; urine and bowel continence $(p=.06))$ at the end of physical activity program. These relationships not reach the level of acceptability $(p<.05)$, probably because of the low sample size.

\section{CONCLUSION}

These results underlined at first the benefits of participation at a training of physical activity for institutionalized older people. At second that also in critical condition, as that of the older people living in residential care facility, the introduction of a relatively simple training may have positive effects on the individual functioning in short time. Finally, our study seems to demonstrate that the relative easiness of application of this kind of programme must be considered carefully for the promotion of positive development in old age.

Exploring involvement in movement of active young older people in order to plan an adequate offer

Magistro Daniele *, Liubicich Monica Emma*, Marco Pianotti*, Candela Filippo**, Rabaglietti Emanuela**, Kratter Gioachino*

*Motor Science Research Center, SUISM (University School of Motor \& Sport Sciences), University of Turin, **Department of Psychology, University of Turin, Italy

\section{INTRODUCTION}

Given the increasing life expectancy in all western society to investigate the group of active young older people is important, not only in order to individuate protective and risk factors of diseases, but also to know the real needs of this population and to plan well structured activities. Successful ageing is likely to be related to healthy lifestyle. That is increasing the amount of daily physical activities may mean improving the wellbeing of senior citizens. The present study is aimed at exploring in a sample of normative young older people: (a) participation in physical activity, (b) preferred time of the day for participating at physical activity (c) level of stress assigned to the movement.

\section{METHODS}

Two hundreds and two young older people (124 men and 78 women; mean age $=66.86$, st.dev. $=5.31)$ participated at the study. They were administered the International Physical Activity Questionnaire (IPAQ). We used descriptive statistical techniques and ANOVA for analyzing the data.

\section{RESULTS}

Only $25 \%$ of young and normative older people practiced a physical activity, mainly in the morning followed by late afternoon, early afternoon and finally evening. There were no gender differences with respect to the time of the day when older people usually practice exercise. Physical activity is perceived, on average, heavier than other types 
of activity (as being involved in chores and or in other leisure activities) regardless the time of the day: a) morning $[\mathrm{F}(1,164)=6.40, p<.012, \eta 2=.04]$; b) afternoon $[\mathrm{F}(1,158)=4.11, p<.044, \eta 2=.02]$; $\mathrm{c})$ evening $[\mathrm{F}(1,166)=$ $3.76, p<.05, \eta 2=.02]$.

\section{CONCLUSION}

In a society that is going to become old or very old, there is a increasingly need of studies addressed to the world of the elderly and aimed at designing interventions that can help in improving the quality of life. Our findings suggested that, in order to facilitate regular physical activity of active groups of seniors, we have to consider not only the kind of physical activity more suitable, but also the efforts that older people perceived moving and the time of the day when these activities are planned.

\section{The comparison of physical fitness and quality of life in Mountaineer aged people}

Maryam Nourshahi ${ }^{1}$, Behrouz Abdoli ${ }^{2}$,Asal Rajaeyan ${ }^{3}$, Hadi Zahedi ${ }^{4}$,Akram Kaviyani ${ }^{5}$

${ }^{1-5}$ Department of Exercise Physiology, Faculty of physical Education and sport Sciences, Shahid Beheshti University, G.C..Evin 198396113, Tehran, Iran

Introduction: Aging leads to gradual decrease in physiological capacities. Faulkner et al 2008 has been proved that the aging process is accompanied with reduction in muscular strength and cardiovascular capacity While exceed body weight and visceral fat. Nourshahi et al 2009 have proposed that increase in body weight can also impair physical functioning, reduce quality of life and adversely affect mental health. In addition, Patersson et al. (2008) have demonstrated that being active in this period is caused to increase hopeful life, quality of life and satisfaction. Since Tehran is the second polluted city in the world and it is a mountainous city with the second highest summit (Damavand) where is located in north of Tehran, in this case the people especially elderly used to go to the mountain for exercising. Therefore, the purpose of this study was to compare physical fitness and quality of life in three elder groups: Mountaineer, Trained and Sedentary.

Methods: Forty three elderly men (mean age $57 / 7 \pm 9 / 3$, height $168 / 5 \pm 12 / 5$ and weight $77 / 3 \pm 21 / 1$ ) were selected randomly through questionnaires that had been distributed in North of Tehran. According to their activeness and type of activity, subjects were divided into three groups of Mountaineer (n: 15), Trained (n: 14) and Sedentary (n: 14). Then quality of life questionnaires (WHOQOL BREF) were administered and their physical fitness factors, which consist of: Body Fat percent, Blood pressure, Heart Rate, BMI, Flexibility, Hand Strength, Static and Dynamic Balance, Reaction time, Modified Balk Test were measured. All data sets were tested for normal distribution using the Kolmogrov-smirnov test. Physiological data were analyzed using one way ANOVA, and the quality of life questionnaire data were analyzed using multivariate analysis of variance (MANOVA).

Results: The results showed that aerobic capacity was significantly higher in mountaineer group in comparison to two other groups $37.2 \%, 15.4 \%$ respectively. There was significant differences among groups in scores of physical health, social relationships, and environment domains. There were any significant differences between groups in psychological domains. Besides, comparing the average of the score of total quality of life in elder mountaineer group (107/73), was better than two other groups (93/07, 80/92 respectively). Conclusion: The finding explains positive effects of regularly physical activity especially mountaineer on both physical fitness and quality of life factors. Therefore, it is recommended to elders to participate in physical activities regularly and mountain climbing too, to increase the quality and length of life.

Key words: physical fitness factors, quality of life, mountaineer, elder people

\section{References:}

Faulkner JA, Davis CS, Mendias CL and Brooks SV. (2008).The aging of elite male athletes: age related changes in performance and skeletal function. . Clin Exp Pharmacol Physiol.18 (6): 501-507.

Nourshahi M, S Ahmadizad, H Nikbakht, MA Heidarnia and E Ernst. (2009). The effects of triple therapy (acupuncture, diet and exercise) on body weight: a randomized, clinical trial. Int j of obes,33, 583-587.

Patterson SL, Rodgers MM, Macko RF, Forrester LW. (2008) Effect of treadmill exercise training on spatial and temporal gait parameters in subjects with chronic stroke: A preliminary report. J Rehabil Res Dev;45(2):221-8.

\section{Social and psychological aspects}

\section{Aspect of lifelong sports policy in Turkey}

Sebahattin Devecioğlu, Yüksel Savucu, Bilal Çoban

School of Physical Education and Sports, Firat University, 23119, Elazı̆̆, Turkey

\section{Introduction}

In every age and every level of sports participation to ensure the individuals will contribute to social develop- 
ment. Also much participation in a sporting environment gives an opportunity to develop sport versatile. Together to conduct an active participation in sport the passive participation will contribute healthy environment. In this sense, community who has multi-sport environment can take up both sports and levels of social development.

\section{Methods}

This study aimed that characterization of lifelong sports policy in Turkey had been examined by using the "documentation method" and evaluated some basic issues by "retrospective method". All the documents and the findings were interpreted by analysis.

\section{Results and discussion}

Turkey's basic functional sports organization is the Ministry of State Youth and Sports General Directorate was established in 1986 by law number of 3289 .

The purpose of this institution is to steer the physical, moral strength and their ability providing physical education, games, gymnastics, and sports activities for citizens and young people in schools, to conduct the services relating to assessment of leisure of youth, to organize the knowledge and skills courses, to take the necessary measures to protect bad habits of young people.

For encourage lifelong participation in sport; the legal arrangements were given in the Constitution of the Republic of Turkey of 58. and 59. substance.

Also in the process of nomination to the European Union regulations regarding sports-related efforts are to take measures.

\section{Conclusion}

In Turkey, lifelong sports participation was provided to various legal regulations in the context of sports federations, clubs and regional sports activities develop in terms of necessary measures taken, the policy goals of the citizens of all ages, gender and socio-economic level of interests and purposes of the sports facilities to provide is seen as.

It had been shown that sports practices for regular and healthy living in Turkey are not yet sufficiently understood in terms of health, fitness, beauty, balance and efficiency. In this regard, the state are need many sports scientists, physical education and sports leaders and their contributions needed and the cooperation of all concerned.

Key Words: Sports, lifelong sports, policy

The comparison of body image between athletes and nonathletes menopausal females

Abdollah Ghasemi. Maryam Momeni

Islamic Azad University/Tehran/Iran

Rehabilitation and Welfare Science University/Tehran/Iran
Introduction: One of the biggest problem of menopausal women is self-concept that can cause a lot of psychological problems for them. The aim of the present study was to compare and contrast the sub-scale of the body image of the athletes and nonathletes menopause females.

Methods: The subjects of the research comprise 60 individuals whose ages are between 43-53. The instruments used to collect data for the dependant variable included a personal information questionnaire and a PSDQ .The PSDQ included the sub-scales of power, endurance, coordination, general health, flexibility, self-esteem, athletic competence, fat, body appearance, body activity and the whole body. The statistical procedures used in this study comprised Kolmogrov- Smirnov and the $\mathrm{T}$ test.

Results and discussion: The statistical results showed that the body image of the athletes in all of sub-scales is significantly better than individuals who were not athletes $(p \leq 0 / 01)$. Thus the result showed that the attitude of the individual in due to their continuous physical activity in that the athletes have got better body images as compared to the nonathletes who have not physical activity.

Conclusion: It seems that participate in physical activity programs have a positive effect on menopausal side effects and can promotes the self -esteem and psychological aspect of body among menopause women.

Key terms: body image, athlete menopause females, nonathlete menopause females

\section{Reference:}

1. Taylor AH, Fox KR. Effectiveness of a primary care exercise referral intervention for changing physical selfperceptions over 9 months. Health Psychol. 2005, Jan;24 (1):11-21

2. Sands R.T .female wheelchair athletes and changes to body Image, Journal of disability, 2000, 413-426(14)

3. Margaret Schneider, Physical Activity and Physical SelfConcept among Sedentary Adolescent Females; an Intervention Study. Psycho Sport Exerc. 2008, Author manuscript

Physical inactivity and related barriers to the practice, in older Brazilians

Sebastião Gobbi, Emerson Sebastião, Eduardo Kokubun, Camila Priscila M. Nakamura, Camila B. Papini, Camila V. L. Teixeira, Lilian T. B. Gobbi

Instituto de Biociências, UNESP-Univ. Estadual Paulista, Laboratório de Atividade Física e Envelhecimento (LAFE) e Núcleo de Atividade Física, Esportes e Saúde (NAFES), Rio Claro-SP, Brazil

\section{Introduction}

There is scientific consensus that regular engangement in physical exercise brings substantial benefits in all dimen- 
sions of living particularly for older people. However, personal or environmental barriers can contribute to dificulty or even to prevent such engagement. This issue is important for policy makers and well being among the older people. Therfore the aim of this study was to analyze the physical inactivity and barriers to the practice of physical activity in older Brazilian people.

\section{Methods}

By means of randomized and stratified by conglomerates technique, a 100 out of 200 census sections in Rio Claro city-Brazil (close to 200 thousands inhabitants) were selected and 1596 people participated for a comprehensive study. In the present study only the data on physical inactivity and barriers provided by older people $(n=359$; $79.2 \pm 7.5$ year-old) were analyzed. Data on physical inactivity were collected by means of the International Physical Activity Questionnaire (IPAQ-Long version). Barriers were assessed by a specific questionnaire based on those reported in the scientific literature for the same purpose.

\section{Results and discussion}

It was found high prevalence of physical inactivity ( $\%$ of people who had been performing less than 150 min per week) in all domains of the IPAQ, with exception to household one (work $=86 \%$; household $=42 \%$; leisure $=$ $80 \%$, and locomotion $=69 \%$ ). Regarding the barriers, the most reported ones in decrescent order were: "Need to rest or relax" (58\%); "Already active enough"(53\%); Fear of injury (44\%); "Feel lazy"(43\%); "No facility nearby" $(42 \%)$.

\section{Conclusion}

Older people living in Brazilian medium size cities present high prevalence of physical inactivity, particularly in work, locomotion and leisure domains. Consistent with such findings they also present high perception of barriers to the practice of physical activity. The results of this study may help public policy makers to better address the question of active lifestyle among older people.

\section{Acknowledgements \\ CNPq; FUNDUNESP; FAPESP.}

\section{Predicting physical exercise in older people through the theory of planned behaviour \\ Bonete, B. \& Sitges, E \\ Miguel Hernández de Elche University, Elche, Spain}

\section{Introduction}

Specialists in the study of predictors of physical exercise, such as Gould and Weinberg (2003), indicate that the behaviour of doing exercise should be dealt with in conceptual methodological way and from a holistic perspective of health. This behaviour is explained through four social cognitive models which have high empirical comsistency: the health belief model, the trans-theory model, the cognitive social theory and the planned behaviour theory. Out of these models, the planned behaviour theory by Ajzen and Madsen (1986) has proved to have the greatest predictive validity for explaining and predicting behaviours related to physical exercise (Symons y Hausenblas, 2005; Hagger, 2006).

\section{Method}

The metanalyses carried out on Planned Behaviour Theory up to present day have been analysed and those that include the older population in their samples are detailed.

\section{Results}

The most relevant results are found to be in line with the findings obtained by Estabrooks and Carron (1999), which indicate that perceived control is the most determining factor in the model, whereas attitude is not directly related to the intention of doing exercise.

\section{Conclusions}

In general, the different research papers analysed confirm that if there is a desire within the older population to initiate or maintain the behaviour of doing exercise, we should focus on increasing perceived control over the factors that encourage doing the activity, such as favouring the opportunity of the action, specifying the time or moment when the behaviour should be carried out and eliminating obstacles that could stop this action form being completed (Kerner et al., 2001; Schutzer, K.A. y Graves, B.S., 2004).

Ajzen, I., \& Madden, T. J. (1986). Prediction of goal directed behavior: Attitudes, intentions, and perceived behavioral control. Journal of Experimental Social Psychology, 22, 453-474.

Estabrooks, P., \& Carron, A. V. (1999). The influence of the group with elderly exercisers. Small Group Research, 30, 438-452.

Gould, D. \& Weinberg, R.S. (2003). Foundations of sports \& exercise psychology, 3rd. ed. (pp. 586). Champaign: Human kinetics publishers.

Hagger, M.S. (2006). Meta-analysis in sport and exercise research: review, recent developments, and recommendations. European Journal of Sport Science, 6, 103-115.

Kerner, M. S., Grossman, A. H., \& Kurrant, A. B. (2001). The theory of planned behavior as related to intention to exercise and exercise behavior. Perceptual \& Motor Skills, 92, 721-731.

Schutzer, K.A. \&Graves, B.S. (2004). Barriers and motivations to exercise in older adults. Preventive medicine, 39, 1056-1061.

Symons, D. \& Hausenblas, H.A. (2005). Elicitation studies and the theory of planned behavior: a systematic review of exercise beliefs. Psychology of Sport \& Exercise, 6, 1-31. 


\section{Geri Olympics programs}

Ted Muilenburg

Department of Health and Human Performance, West Virginia State University, Institute,WV HTM406@AOL.COM

INTRODUCTION: The Geri Olympics Programs were introduced 23 years ago as a way of improving the quality of life of nursing home residents (nursing home resident athletes) through competitive sports. Wellness, health, active living, social engagement, involvement with children and the community have all been identified as benefits of Geri Olympics. Nursing homes and the residents are often over looked in most communities - they are seen by some segments as going to there to die. Nursing care facilities provide excellent care and rehabilitation. The Geri Olympics have provided additional support in managing some of the non-medical issues such as loneliness, hopelessness and boredom. Through cooperation and collaboration with nursing home residents, health care professionals, university students and faculty, and community leaders programs have evolved that in 2010 served over 300 nursing home residents. In addition to events in West Virginia Geri Olympics have expanded to include a similar program in Prague through a Memo of Understanding between Charles University and West Virginia State University.

METHODS: Geri Olympics have evolved over 23 years through cooperation with health care professionals, nursing home residents, and host of other people. Events such as candle blowing and clothes pin stacking were added as a result of recommendation by representatives from Charles University and are designed to improve pulmonary function and fine motor coordination. Growth and expansion of programs continues to grow at a steady rate because staff are responsive to suggestions and ideas generated by cooperative partners and the nursing home resident.

RESULTS AND DISCUSSION: In 2010200 nursing home resident athletes participated in the Geri Olympics from 25 nursing care facilities. Over 200 volunteers from 21 different organizations served as volunteers and "cheer leaders". Many of the volunteers were children and youth who came to cheer and help with the competition, providing an intergenerational component. Geri Olympics have become a community event that promotes the best interests of nursing home residents and advocating for their quality of life and importance.

CONCLUSIONS: Geri Olympics will continue in the future to promote the "best interests" of the nursing home resident and advocate for their quality of life through competitive sports, active living and wellness. Sustainability is accomplished through expanded cooper- ation and collaboration with a wide variety of partners at home and abroad who have an interest in and value seniors.

Key words: wellness, seniors, active living, nursing home, competitive sports

The Effect of Eight weeks Hydrotherapy on the Mental Health of Men personnel 40-50 years of oil company Nasrolahi $\mathbf{S}^{1}$ Zarghami $\mathbf{M}^{2}$-Masoud $\mathbf{N}^{3}$-Valizadeh $\mathbf{R}^{4}$ 1-national iranian south oil company portal, Iran

2,3-Faculty of physical Education \& Sport Science Shahid Chamran University-Ahwaz - Iran

4-Faculty of physical Education \& Sport Science of Islamic Azad University, Omidiyeh Branch, Iran

\section{Introduction}

The purpose of conducting this study is to consider the effect of 8 weeks hydro-therapy practices on mental health, depression, anxiety. Physical complaints and disorder in social function of employees of the National company for south oil-bearing areas.

\section{Methods}

The present study is semi-experimental type performing in field and using experimental and control group. Celburg and healer general health questionnaire GHQ28 has been used in this study. Also by using hydrotherapy practices test group was practiced in swimming pool. The statistical society for the study is all the 40 50 years old male employees in the National company for south oil-bearing areas that after calculating mental health point with one and half criterion deviation higher than average, 80 people selected in randomly among them and then these people divided into 2 experimental and control groups of 40 people. Analyzing information was performed by computer and using spss software and statistical method of MANOVA

\section{Results and discussion}

Considering the study findings showed that hydro-therapy practices has a positive effect on mental health, physical complaints anxiety and depression, while it has no effect on disorder in social function. since level of hormones that such as norepinephrine, serotonin and dopamine is low in body of some one who suffer than depression and anxiety. seem that hydro-therapy exercise could increase level of this hormones.(1)

\section{Conclusion}

Suggest to person who suffer than depression, anxiety that for decrease depression and anxiety and another disorders do hydro-therapy exercise in swimming pool 
because that when we do exercise in water we have at least pressure on our joints in compare with do exercise in out of water.(2)

\section{References}

1-Gaszkow ska M.(2004)"Effect of exercise on anxiety and mood psychiatrpoblish",38(4)611-20

2-Netz Y,Wumeny J,Backer BJ,Tenenbaum G(2005).Physical activity and psychological well-being in advanced age: A meta analysis of intervention studies.Psychol Aging 20 (2)272-284.

Frequency of knowledge of performance in aquisition of the basketball free throw in old age: movement pattern and scoring relationship

Santos, S.; Nunes, M.E.S.; Fonseca, M.C.O.; Franzoni, M.M. Laboratory of Motor Behavior

School of Physical Education and Sport

University of Sao Paulo, Brazil

\section{Introduction}

Motor learning researchers have been devoted to examine factors that can affect the acquisition of a motor skill with special emphasis to the effect of feedback, as it has been recognized, along with the practice, as crucial factors to improve performance. It is well known that Knowledgement of Performance (KP) function is to guide the learner attention to critical aspects of the movement pattern. Considering that in old age, attention and memory can become a problem; the objective of this study was to investigate the effect of frequency of KP $(100 \%, 66 \%$, and $33 \%$ ) during the acquisition of basketball free throw in old age, particularly, whether there is a relationship between movement pattern and scoring.

\section{Method}

The task goal was to perform a basketball free throw. Sixty active individuals (men and women) aged from 60 to 69 years old were divided into 3 experimental groups, which received $100 \%, 66 \%$, and $33 \%$ of KP frequency, during 90 trials in 3 practice sessions. Volunteers were also asked to perform a retention and transfer tests after $24 \mathrm{~h}$. During the acquisition phase, volunteers received $\mathrm{KP}$ about the movement pattern of the previous attempt from a qualitative hierarchical checklist of the free throw (14 items). Sessions were recorded in order to confirm whether volunteers were able to score throughout sessions.

\section{Results}

ANOVA indicated that the group which received KP in $66 \%$ of total trials showed superior performance in comparison to both, $100 \%$ and $33 \%$ groups. Correlation analyses also indicated that there was a significant relationship between movement pattern and scoring in all groups, but the relationship was weaker in the group which received $100 \% \mathrm{KP}$.

\section{Discussion}

All individuals showed an improved performance in the retention and transfer tests. But the $66 \% \mathrm{KP}$ group was superior in both qualitative (movement pattern) and quantitative (score) measurements throughout the trials. For one hand, the checklist used in this study seemed to appropriately evaluate individuals movement pattern, but the fact that the relationship between movement pattern and scoring was weaker in the $100 \% \mathrm{KP}$ group, could be argued in terms of the performance variability.

\section{Self-selected pace walking affects frontal eeg asymmetry} and mood in the elderly

Tobias Vogt, Stefan Schneider, Vera Brümmer, Heiko K Strueder

Institute of Movement and Neurosciences, German Sport University Cologne

\section{Introduction}

Recently the connection of physical exercise and changes in brain cortical activity [1] as well as links to mood has been discussed [4]. However, due to inappropriate study designs for the elderly (i.e. intensity) most studies were limited to samples of young, healthy and predominantly physically active individuals $[2,3]$. Though in times of an aging society, where the benefits of physical exercise gain interest, it seems increasingly necessary to investigate the connection of psycho-physiological elements of exercise. According to previous research we hypothesised that physical exercising at self-selected pace in the elderly increases left frontal brain activity (pronounced in alpha) that is associated with a positive change in mood.

\section{Methods}

We recorded electroencephalographic (EEG) data on two frontal positions (Fp1, Fp2) as well as actual state of mood before and after walking (45 to $60 \mathrm{~min}$ ). The equation (right-left)/(right+left) served to calculate frontal asymmetry as a marker of approach-related emotions [5].

\section{Results and discussion}

As key findings we found (1) a shift within the alpha-1 $(7.5-10.0 \mathrm{~Hz})$ activation towards right frontal brain areas as well as (2) an increase in mood (perceived 
physical health and fitness) after walking. Referring to the model of frontal asymmetry with respect to the mood findings, the increase $(p<.05)$ in the alpha-1 activation can be associated with approach-related emotions.

\section{Conclusion}

In conclusion there is reason to speculate, that common physical exercising affects a general well-being in the elderly; displayed in both, subjective perception and objective physiological parameters, such as brain activity. In this pilot study we were able to demonstrate this by a comparatively simple and economic method.

\section{References}

[1] JB Crabbe, RK Dishman, Brain electrocortical activity during and after exercise: a quantitative synthesis. Psychophysiol. 41(4) (2004) 563-574.
[2] P Ekkekakis, EE Hall, LM Van Landuyt, SJ Petruzzello, Walking in (affective) circles: can short walks enhance affect? J Behav Med. 23(3) (2000) 245-275.

[3] S Schneider, CD Askew, J Diehl, A Mierau, J Kleinert, T Abel, H Carnahan, HK Strueder, EEG activity and mood in health orientated runners after different exercise intensities. Physiol Behav. 96(4-5) (2009) 709-16.

[4] S Schneider, V Bruemmer, T Abel, CD Askew, HK Strueder, Changes in brain cortical activity measured by EEG are related to individual exercise preferences. Physiol Behav. 98(4) (2009) 447-52.

[5] DJ Schutter, AD Weijer, JD Meuwese, B Morgan, JV Honk, Interrelations between motivational stance, cortical excitability, and the frontal electroencephalogram asymmetry of emotion: A transcranial magnetic stimulation study. Hum Brain Mapp. 29(5) (2008) 574-580. 